Kyushu J. Math.

Vol. 54, 2000, pp. 7-37

\title{
CROSS RATIO VARIETIES FOR ROOT SYSTEMS II: \\ THE CASE OF THE ROOT SYSTEM OF TYPE $E_{7}$
}

\author{
Jiro SEKIGUCHI
}

(Received 6 May 1998 and revised 13 January 1999 and 14 September 1999)

\section{Introduction}

We introduced the notion of cross ratio varieties for root systems in [14], generalizing the construction of Naruki's cross ratio variety $C$ in [9], which is a smooth compactification of the moduli space of cubic surfaces. In our formulation, $C$ can be regarded as one of the cross ratio varieties related to the root system of type $E_{6}$. The variety $C$ has some nice properties which are explained later in this introduction. In the case of the root system of type $E_{7}$, we obtain three kinds of cross ratio varieties. We focus our attention on one, denoted $C_{E_{7}}$ in this article. This variety $C_{E_{7}}$ can be regarded as an analogue of Naruki's cross ratio variety in the sense that it is regarded as a moduli space of marked Del Pezzo surfaces of degree two. Our subject is related to studies of 27 lines on cubic surfaces and 28 bitangents to plane quartic curves, classical (cf. $[2,8])$ and modern (cf. $[21,22]$ ).

Before explaining the present study, we review the definition of $C_{E_{7}}$ briefly. Let $\mathbf{P}(2,7)$ be the configuration space of seven points in the complex projective plane $\mathbf{P}^{2}$, namely, the quotient space of $\tilde{\mathbf{P}}(2,7)$ under the $P G L(3)$-action, where

$$
\begin{aligned}
\tilde{\mathbf{P}}(2,7)=\left\{\left(P_{1}, \ldots, P_{7}\right) ;\right. & \text { (a) } P_{i} \in \mathbf{P}^{2}(i=1,2, \ldots, 7), \\
& \text { (b) } P_{i} \neq P_{j}(i \neq j), \\
& \text { (c) No line passes through three of } \left.P_{1}, \ldots, P_{7}\right\} .
\end{aligned}
$$

Moreover, we introduce an open subset $\mathbf{P}_{0}(2,7)$ of $\mathbf{P}(2,7)$ defined as the quotient space of $\tilde{\mathbf{P}}_{0}(2,7)$ under the $P G L(3)$-action, where

$\tilde{\mathbf{P}}_{0}(2,7)=\left\{\left(P_{1}, \ldots, P_{7}\right) \in \tilde{\mathbf{P}}(2,7) ;\left(\right.\right.$ d) No conic passes through six of $\left.P_{1}, \ldots, P_{7}\right\}$.

Note that $\mathbf{P}_{0}(2,7)$ admits a biregular $W\left(E_{7}\right)$-action, though $\mathbf{P}(2,7)$ does not, where $W\left(E_{7}\right)$ is the Weyl group of type $E_{7}$. The variety $C_{E_{7}}$ is defined as a compactification of $\mathbf{P}_{0}(2,7)$ obtained by cross ratios for the root system. The $W\left(E_{7}\right)$-action on $\mathbf{P}_{0}(2,7)$ extends to $C_{E_{7}}$ in a natural manner. 
We now recall basic properties of Naruki's cross ratio variety $C$ (cf. $[9,13])$ :

(i) $C$ is a compactification of $\mathbf{P}_{0}(2,6)$, which is the configuration space of six points defined similarly to $\mathbf{P}_{0}(2,7)$.

(ii) $C$ is non-singular.

(iii) $C$ admits a biregular $W\left(E_{6}\right)$-action, where $W\left(E_{6}\right)$ is the Weyl group of type $E_{6}$.

(iv) The complement of $\mathbf{P}_{0}(2,6)$ in $C$ is a divisor with normal crossings only. It is the union of seventy six non-singular hypersurfaces of $C$ and they are divided into two $W\left(E_{6}\right)$-orbits. Moreover, such a hypersurface is described in terms of a subset of the root system of type $E_{6}$.

Since the construction of $C_{E_{7}}$ is similar to that of $C$, we are led to the following problem.

\section{Problem.}

(i) Is the variety $C_{E_{7}}$ non-singular?

(ii) Is the $W\left(E_{7}\right)$-action on $C_{E_{7}}$ biregular?

(iii) Is $C_{E_{7}}-\mathbf{P}_{0}(2,7)$ a divisor of $C_{E_{7}}$ with normal crossings only? Determine the irreducible components of $C_{E_{7}}-\mathbf{P}_{0}(2,7)$ and describe them in terms of subsets of the root system $\Delta=\Delta\left(E_{7}\right)$ of type $E_{7}$.

Bearing this problem in mind, I focus my attention on the determination of irreducible components of $S_{E_{7}}=C_{E_{7}}-\mathbf{P}_{0}(2,7)$. We have already proposed an idea to describe the irreducible components in terms of subsets of the root system of type $E_{7}$ and found some of them in [14]. There we introduced the subvariety $Y_{\Delta, D_{4}}\left(\Delta_{1}\right)$ of $C_{E_{7}}$ corresponding to each irreducible subroot system $\Delta_{1}$ of $\Delta$. As its basic property, $Y_{\Delta, D_{4}}\left(\Delta_{1}\right)$ itself is also a cross ratio variety for a certain root system. If $\Delta_{1}$ is of type $A_{1}$ (respectively $\left.A_{2}, A_{3}, A_{6}\right), Y_{\Delta, D_{4}}\left(\Delta_{1}\right)$ is called a hypersurface of the 1 st kind, (respectively the 2nd kind, 3rd kind, 4th kind) and these four kinds of hypersurfaces are contained in $S_{E_{7}}$. But $Y_{\Delta, D_{4}}\left(\Delta_{1}\right)$ is not contained in $S_{E_{7}}$ in general (for example, the case where $\Delta_{1}$ is of type $E_{6}$ ). Since the above four kinds of hypersurfaces are the only subvarieties of $C_{E_{7}}$ which are of the form $Y_{\Delta, D_{4}}\left(\Delta_{1}\right)$ and which are also hypersurfaces contained in $S_{E_{7}}$, it is interesting to study whether $S_{E_{7}}$ is the union of the hypersurfaces of the 1st-4th kinds or not (see [14, Conjecture 4.14]).

The main purpose of this article is to study the structure of hypersurfaces in $C_{E_{7}}$ of the forms $Y_{\triangle, D_{4}}\left(\Delta_{1}\right)$ in the case where the type of $\Delta_{1}$ is one of the following: $A_{1}, A_{2}, A_{3}, A_{6}, E_{6}$. There are two reasons why we treat the case $E_{6}$ in addition to the cases $A_{1}, A_{2}, A_{3}, A_{6}$. One is that if $\Delta_{1}$ is an irreducible proper subroot system of $\Delta$ such that $\Delta_{1}$ is not of type $A_{7}$, then $Y_{\triangle, D_{4}}\left(\Delta_{1}\right)$ coincides with or is contained in the subvariety $Y_{\Delta, D_{4}}\left(\Delta_{1}^{\prime}\right)$ corresponding to a subroot system $\Delta_{1}^{\prime}$ whose type is one of 
$A_{1}, A_{2}, A_{3}, A_{6}, E_{6}$ and that if $\Delta_{1}$ is of type $A_{7}, Y_{\Delta, D_{4}}\left(\Delta_{1}\right)$ coincides with $C_{E_{7}}$ itself. The other is that the subvariety $Y_{\triangle, D_{4}}\left(\Delta_{1}\right)$ corresponding to a root system of type $E_{6}$ is interesting in its own sake; each point represents a seven points set in $\mathbf{P}^{2}$ related with a special flex of a non-singular plane quartic curve (cf. [22]). Partial results were already announced in [14, §4, Remark 4.13 and (4.15.1)-(4.15.11)].

We explain the contents of this article.

We begin Section 2 by reviewing the previous article [14]. In particular, a description of hypersurfaces of the 1st-4th kinds in terms of root systems is given and the intersection relation among them is studied. In the last part of this section, in Theorem 1, we describe the intersection relation between hypersurfaces of the 4th kind and other hypersurfaces. This is supplementary to Theorem 4.7 in [14] (see also [14, Remark 4.13]).

In Section 3, we study the structure of the intersections of hypersurfaces of the 1st-4th kinds. Let $Y$ and $Y^{\prime}$ be such hypersurfaces. The main purpose in this section is to determine the structure of $Y \cap Y^{\prime}$. If one of them is of the $2 \mathrm{nd}$, 3rd or 4th kind, we find that their intersection is isomorphic to a Terada model or a product of Terada models. Thus it is easy to treat such cases. The results are given in Sections 3.1-3.3. Next we study the case where both of them are of the 1st kind. More generally, we study the intersections of several hypersurfaces of the 1 st kind. We write $Y_{\gamma}=Y_{\Delta, D_{4}}(\{ \pm \gamma\})$, which is of the 1st kind, where $\gamma \in \Delta$. As we proved in [14], $Y_{\gamma}$ is isomorphic to the cross ratio variety $C\left(\Delta\left(D_{6}\right),\left\{A_{3}^{(1)}, D_{4}\right\}\right)$ for the root system $\Delta\left(D_{6}\right)$ of type $D_{6}$. In Section 3.4, we study subvarieties of the form $Y_{\Delta\left(D_{6}\right), D_{4}}\left(\Delta^{\prime}\right)$ in $Y_{\gamma}$ for subroot systems $\Delta^{\prime}$ of $\Delta\left(D_{6}\right)$ whose types are $A_{1}+\cdots+A_{1}$. From the definition, $Y_{\Delta\left(D_{6}\right), D_{4}}\left(\Delta^{\prime}\right)$ is equal to the intersection of hypersurfaces of the 1st kind. Among others, we explain two interesting cases. The first case is the intersection $Y_{\gamma} \cap Y_{\gamma^{\prime}}$ for mutually orthogonal roots $\gamma, \gamma^{\prime}$ of $\Delta$. In this case, $Y_{\gamma} \cap Y_{\gamma^{\prime}}$ is embedded into $\left(\mathbf{P}^{1}\right)^{57}$ and there is a natural birational map of $Y_{\gamma} \cap Y_{\gamma^{\prime}}$ onto $C\left(\Delta\left(D_{5}\right),\left\{A_{3}^{(1)}, D_{4}\right\}\right) \simeq C$, Naruki's cross ratio variety. The second case is the intersection $Y=\bigcap_{j=1}^{4} Y_{\beta_{j}}$, where $\beta_{1}, \beta_{2}, \beta_{3}, \beta_{4} \in \Delta$ are mutually orthogonal. Then $Y$ is isomorphic to the projective variety $\tilde{Z}$ constructed as follows. Let $Z$ be a subvariety of $\mathbf{P}^{2} \times \mathbf{P}^{2}$ defined by

$$
\left\{\left(\left(\xi_{1}: \xi_{2}: \xi_{3}\right),\left(\eta_{1}: \eta_{2}: \eta_{3}\right)\right) \in \mathbf{P}^{2} \times \mathbf{P}^{2} ; \xi_{1} \eta_{1}=\xi_{2} \eta_{2}=\xi_{3} \eta_{3}\right\}
$$

Then $\tilde{Z}$ is the variety obtained by blowing up $Z$ at the three points

$$
\begin{gathered}
((0: 1:-1),(1: 0: 0)), \quad((1: 0:-1),(0: 1: 0)), \\
((1:-1: 0),(0: 0: 1)) .
\end{gathered}
$$


The statements [14, (4.15.1)-(4.15.11)] are easy consequences of the results in Section 3.

In Section 4 , we treat the case $\Delta_{1}=\Delta\left(E_{6}\right)$. There are in total 28 hypersurfaces in $C_{E_{7}}$ which are transformed to $Y_{\Delta, D_{4}}\left(\Delta\left(E_{6}\right)\right)$ under $W\left(E_{7}\right)$-action. Different from the four kinds of hypersurfaces, $Y_{\Delta, D_{4}}\left(\Delta\left(E_{6}\right)\right)$ is not contained in $S_{E_{7}}$. It is easy to see (cf. Lemma 6) that $Y_{\triangle, D_{4}}\left(\Delta\left(E_{6}\right)\right.$ ) is isomorphic to the cross ratio variety $C\left(\Delta\left(E_{6}\right),\left\{A_{3}, D_{4}\right\}\right)$ for the root system $\Delta\left(E_{6}\right)$. The purpose of this section is to characterize $Y_{\Delta, D_{4}}\left(\Delta\left(E_{6}\right)\right)$ in terms of configurations of seven points. Given seven points $P_{1}, \ldots, P_{7}$ of $\mathbf{P}^{2}$ in general position, there is a unique cubic curve $C$ in $\mathbf{P}^{2}$ passing through $P_{1}, \ldots, P_{7}$ such that one of them, say $P_{7}$, is a double point (cf. [7, 8]). We consider a condition on the seven points $P_{1}, \ldots, P_{7}$ so that the double point $P_{7}$ becomes a cusp. By this condition, we define a hypersurface $Y\left(\left\{P_{1}, \ldots, P_{7}\right\} ; P_{7}\right)$ of the configuration space $\mathbf{P}_{0}(2,7)$. Then we show a characterization of $Y_{\Delta, D_{4}}\left(\Delta\left(E_{6}\right)\right)$ in Theorem 2, which states that the closure of $Y\left(\left\{P_{1}, \ldots, P_{7}\right\} ; P_{7}\right)$ in $C_{E_{7}}$ coincides with $Y_{\Delta, D_{4}}\left(\Delta\left(E_{6}\right)\right)$. There is an alternative construction of $Y_{\Delta, D_{4}}\left(\Delta\left(E_{6}\right)\right)$ by using a $W\left(E_{7}\right)$-invariant polynomial of degree 10 (cf. [15]).

Closing this introduction, we mention recent progresses on the subject treated in this article.

(1) After I completed this article, I was informed by J. Matsuzawa that he and I. Naruki are solving the Problem stated earlier as well as [14, Conjecture 4.14], which states that $S_{E_{7}}$ is the union of the hypersurfaces of $1 \mathrm{st}-4$ th kinds.

(2) Though we treat the cross ratio varieties $C, C_{E_{7}}, \mathbf{P}(2,7)$ and others on the complex number field, these spaces are defined over the real number field. The real loci of $C$ and $C_{E_{7}}$ are related with arrangements of six lines and those of seven lines on the real projective plane. Classifications of arrangements of six lines and seven lines in general position on the real projective plane are already established (cf. [5]). Our treatment of $C$ and $C_{E_{7}}$ naturally leads us to interprete the classifications in terms of root systems of types $E_{6}, E_{7}$. For the details and related topics, see $[3,4,17,18,19,20]$.

\section{Hypersurfaces of $C_{E_{7}}$ corresponding to subroot systems}

Throughout this article, we basically follow the notation in [14]. Before introducing the cross ratio variety $C_{E_{7}}$ (which is denoted $C\left(\Delta\left(E_{7}\right), D_{4}\right)$ in [14]), we recall some basic facts on Terada models. Let $\mathbf{T}_{n}$ be the $n$-dimensional Terada model which is obtained by blowing up $\mathbf{P}^{n}$ (cf. [11, 16, 24]).

Let $\tilde{E}$ be an $(n+1)$-dimensional vector space over $\mathbf{R}$ with a basis 
$\left\{\varepsilon_{1}, \ldots, \varepsilon_{n}, \varepsilon_{n+1}\right\}$. Then the root system $\Delta\left(A_{n}\right)$ of type $A_{n}$ consists of the roots $\varepsilon_{i}-\varepsilon_{j}$ $(i, j=1, \ldots, n, n+1, i \neq j)$. If $E$ is the vector space spanned by $\varepsilon_{i}-\varepsilon_{j}(i \neq j)$, then $\Delta\left(A_{n}\right)$ is, in fact, a root system on $E$. It is shown in [16] that the cross ratio variety $C\left(\Delta\left(A_{n+2}\right), A_{3}\right)$ introduced in [14] is $W\left(A_{n+2}\right)$-equivariantly isomorphic to $\mathbf{T}_{n}$, where $W\left(A_{n+2}\right)$ is the Weyl group of type $A_{n+2}$. The following lemma is shown in [14, Proposition 2.3 and its corollary].

LEMMA 1. Let $\Delta_{0}$ be a subroot system of type $A_{k}(k \leq n+1)$ in $\Delta\left(A_{n+2}\right)$.

(0) If $k=n+1$, then $Y_{\Delta\left(A_{n+2}\right), A_{3}}\left(\Delta_{0}\right) \simeq \mathbf{T}_{n}$.

(i) If $k=1, n$, then $Y_{\Delta\left(A_{n+2}\right), A_{3}}\left(\Delta_{0}\right) \simeq \mathbf{T}_{n-1}$.

(ii) If $1<k<n$, then $Y_{\Delta\left(A_{n+2}\right), A_{3}}\left(\Delta_{0}\right) \simeq \mathbf{T}_{k-1} \times \mathbf{T}_{n-k}$.

(iii) $Y_{\Delta\left(A_{n+2}\right), A_{3}}\left(\Delta_{0}^{\perp}\right)=Y_{\Delta\left(A_{n+2}\right), A_{3}}\left(\Delta_{0}\right)$.

If $J=\left\{i_{1}, \ldots, i_{k}\right\}$ is a subset of $\{1, \ldots, n, n+1\}$, we define a subroot system $\Delta(J)$ in $\Delta\left(A_{n}\right)$ which consists of roots of the form $\varepsilon_{i_{p}}-\varepsilon_{i_{q}}(p, q=1, \ldots, k, p \neq q)$. From the definition, each irreducible subroot system of $\Delta\left(A_{n}\right)$ can be written in this form. For simplicity, we write $\mathbf{T}_{n}(J)$ or $\mathbf{T}_{n}\left(i_{1} \ldots i_{k}\right)$ instead of $Y_{\Delta\left(A_{n+2}\right), A_{3}}(\Delta(J))$. Note that

$$
\mathbf{T}_{n}(J) \simeq \mathbf{T}_{k-1} \times \mathbf{T}_{n-k} .
$$

Moreover, we find from Lemma 1(iii) that if $n$ is odd and if

$$
\left\{i_{1}, \ldots, i_{k}, i_{k+1}\right\} \cup\left\{j_{1}, \ldots, j_{n-k+1}, j_{n-k+2}\right\}=\{1, \ldots, n+2, n+3\},
$$

then

$$
\mathbf{T}_{n}\left(i_{1} \ldots i_{k} i_{k+1}\right)=\mathbf{T}_{n}\left(j_{1} \ldots j_{n-k+1} j_{n-k+2}\right) .
$$

We now recall the definition of the root system $\Delta\left(E_{7}\right)$ of type $E_{7}$. We always denote it by $\Delta$ for simplicity. Let $\tilde{E}$ be an inner product space of dimension 8 with an orthonormal basis $\left\{\varepsilon_{j} ; 1 \leq j \leq 8\right\}$ with respect to an inner product $\langle\cdot, \cdot\rangle$ and let $E$ be its linear subspace orthogonal to $\varepsilon_{7}+\varepsilon_{8}$. As in $[14, \S 4]$, we define the following 63 vectors of $E$ :

$$
\begin{cases}\gamma_{1}=\varepsilon_{7}-\varepsilon_{8} & \\ \gamma_{j}=\varepsilon_{j-1}-\gamma_{0}+\gamma_{1}, & 1<j<8 \\ \gamma_{1 j}=-\varepsilon_{j-1}+\gamma_{0}, & 1<j<8 \\ \gamma_{j k}=\varepsilon_{j-1}-\varepsilon_{k-1}, & 1<j<k<8 \\ \gamma_{1 j k}=-\varepsilon_{j-1}-\varepsilon_{k-1}, & 1<j<k<8 \\ \gamma_{i j k}=-\varepsilon_{i-1}-\varepsilon_{j-1}-\varepsilon_{k-1}+\gamma_{0}, & 1<i<j<k<8,\end{cases}
$$


where

$$
\gamma_{0}=\frac{1}{2} \sum_{j=1}^{8} \varepsilon_{j}-\varepsilon_{8} .
$$

The totality $\Delta$ of $\pm \gamma_{j}, \pm \gamma_{j k}, \pm \gamma_{i j k}$ forms a root system of type $E_{7}$ (cf. [1]).

Remark 1. A geometric meaning of the description of roots $\gamma_{j}, \gamma_{j k}, \gamma_{i j k}$ is given in [23].

As a fundamental set of roots of $\Delta$, we take

$$
\begin{gathered}
\alpha_{1}=\gamma_{12}, \quad \alpha_{2}=\gamma_{123}, \quad \alpha_{3}=\gamma_{23}, \quad \alpha_{4}=\gamma_{34}, \\
\alpha_{5}=\gamma_{45}, \quad \alpha_{6}=\gamma_{56}, \quad \alpha_{7}=\gamma_{67} ;
\end{gathered}
$$

the corresponding Dynkin diagram is

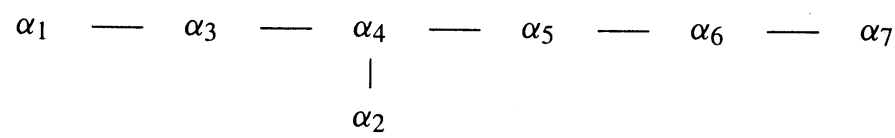

We denote by $\Delta^{+}$the set of positive roots in $\Delta$. It is easy to see that $\Delta^{+}$consists of $\gamma_{i}, \gamma_{i j}, \gamma_{i j k}$. Let $s_{i}, s_{i j}, s_{i j k}$ be the reflections with respect to $\gamma_{i}, \gamma_{i j}, \gamma_{i j k}$, respectively. The group generated by $s_{i}, s_{i j}, s_{i j k}$ for all $i, j, k$ is the Weyl group $W\left(E_{7}\right)$ of type $E_{7}$. In the sequel, we frequently identify $W\left(A_{6}\right) \simeq S_{7}$ (respectively the Weyl group $W\left(E_{6}\right)$ of type $\left.E_{6}\right)$ with the subgroup of $W\left(E_{7}\right)$ generated by $s_{i j}$ (respectively $s_{i j}, s_{i j k}$ $(i, j, k<7))$. Using the 63 positive roots introduced above, we define linear functions on $E$ by

$$
h_{j}=\left\langle\gamma_{j}, t\right\rangle, \quad h_{j k}=\left\langle\gamma_{j k}, t\right\rangle, \quad h_{i j k}=\left\langle\gamma_{i j k}, t\right\rangle,
$$

for $t \in E$.

We briefly review the definition of the configuration space of seven points in $\mathbf{P}^{2}$ which we denote by $\mathbf{P}(2,7)$. We first define the vector space $M_{3,7}$ of $3 \times 7$ matrices. Then $M_{3,7}$ admits $(G L(3) \times G L(7))$-action in a natural manner. Let $T_{G L(7)}$ be the maximal torus of $G L(7)$ consisting of diagonal matrices. Let $D_{i j k}(X)$ be the determinant of the $3 \times 3$ matrix consisting of the $(i, j, k)$ th column vectors of $X \in M_{3,7}$. If $M_{3,7}^{\prime}$ is the subset of $M_{3,7}$ consisting of $X$ with

$$
D_{i j k}(X) \neq 0 \quad \forall(i, j, k)(i<j<k),
$$


we denote by $\mathbf{P}(2,7)$ the quotient of $M_{3,7}^{\prime}$ by the action $G L(3) \times T_{G L(7)}$. It is possible to choose as a representative of any element of $\mathbf{P}(2,7)$ a matrix in the form

$$
X=\left(\begin{array}{ccccccc}
1 & 0 & 0 & 1 & 1 & 1 & 1 \\
0 & 1 & 0 & 1 & x_{1} & x_{2} & x_{3} \\
0 & 0 & 1 & 1 & y_{1} & y_{2} & y_{3}
\end{array}\right)
$$

Therefore $\mathbf{P}(2,7)$ can be regarded as a Zariski open subset of $\mathbf{C}^{6}$ by the correspondence

$$
\left(\begin{array}{ccccccc}
1 & 0 & 0 & 1 & 1 & 1 & 1 \\
0 & 1 & 0 & 1 & x_{1} & x_{2} & x_{3} \\
0 & 0 & 1 & 1 & y_{1} & y_{2} & y_{3}
\end{array}\right) \rightarrow\left(x_{1}, x_{2}, x_{3}, y_{1}, y_{2}, y_{3}\right) .
$$

In fact, $\mathbf{P}(2,7)$ is identified with $\mathbf{C}^{6}-S_{0}\left(A_{6}\right)$, where $S_{0}\left(A_{6}\right)$ is the union of the 28 hypersurfaces below:

$$
\begin{gathered}
x_{i}=0, \quad x_{i}-1=0, \quad y_{i}=0, \quad y_{i}-1=0, \quad x_{i}-x_{j}=0, \quad y_{i}-y_{j}=0, \\
x_{i}-y_{i}=0, \quad x_{i} y_{j}-x_{i} y_{j}=0, \quad\left(1-x_{i}\right)\left(1-y_{j}\right)-\left(1-x_{j}\right)\left(1-y_{i}\right)=0, \\
\varphi_{1}\left(x_{1}, x_{2}, x_{3}, y_{1}, y_{2}, y_{3}\right)=\operatorname{det}\left(\begin{array}{ccc}
1 & 1 & 1 \\
x_{1} & x_{2} & x_{3} \\
y_{1} & y_{2} & y_{3}
\end{array}\right)=0 .
\end{gathered}
$$

We introduce the following seven birational transformations $\sigma_{0}, \sigma_{1}, \ldots, \sigma_{6}$ :

$$
\left\{\begin{array}{lll}
\sigma_{0}:\left(x_{1}, x_{2}, x_{3}, y_{1}, y_{2}, y_{3}\right) & \rightarrow & \left(\frac{1}{x_{1}}, \frac{1}{x_{2}}, \frac{1}{x_{3}}, \frac{1}{y_{1}}, \frac{1}{y_{2}}, \frac{1}{y_{3}}\right) \\
\sigma_{1}:\left(x_{1}, x_{2}, x_{3}, y_{1}, y_{2}, y_{3}\right) & \rightarrow & \left(\frac{1}{x_{1}}, \frac{1}{x_{2}}, \frac{1}{x_{3}}, \frac{y_{1}}{x_{1}}, \frac{y_{2}}{x_{2}}, \frac{y_{3}}{x_{3}}\right) \\
\sigma_{2}:\left(x_{1}, x_{2}, x_{3}, y_{1}, y_{2}, y_{3}\right) & \rightarrow & \left(y_{1}, y_{2}, y_{3}, x_{1}, x_{2}, x_{3}\right) \\
\sigma_{3}:\left(x_{1}, x_{2}, x_{3}, y_{1}, y_{2}, y_{3}\right) & \longrightarrow & \left(x_{1}^{\prime}, x_{2}^{\prime}, x_{3}^{\prime}, y_{1}^{\prime}, y_{2}^{\prime}, y_{3}^{\prime}\right) \\
\sigma_{4}:\left(x_{1}, x_{2}, x_{3}, y_{1}, y_{2}, y_{3}\right) & \longrightarrow & \left(\frac{1}{x_{1}}, \frac{x_{2}}{x_{1}}, \frac{x_{3}}{x_{1}}, \frac{1}{y_{1}}, \frac{y_{2}}{y_{1}}, \frac{y_{3}}{y_{1}}\right) \\
\sigma_{5}:\left(x_{1}, x_{2}, x_{3}, y_{1}, y_{2}, y_{3}\right) & \longrightarrow & \left(x_{2}, x_{1}, x_{3}, y_{2}, y_{1}, y_{3}\right) \\
\sigma_{6}:\left(x_{1}, x_{2}, x_{3}, y_{1}, y_{2}, y_{3}\right) & \longrightarrow & \left(x_{1}, x_{3}, x_{2}, y_{1}, y_{3}, y_{2}\right),
\end{array}\right.
$$

where

$$
x_{j}^{\prime}=\frac{x_{j}-y_{j}}{1-y_{j}}, \quad y_{j}^{\prime}=\frac{y_{j}}{y_{j}-1}, \quad j=1,2,3 .
$$


Let $W_{7}$ be the group generated by $\sigma_{0}, \sigma_{1}, \ldots, \sigma_{6}$ and let $p_{W\left(E_{7}\right)}: W\left(E_{7}\right) \longrightarrow W_{7}$ be the surjective homomorphism defined by

$$
s_{12} \longrightarrow \sigma_{1}, \quad s_{123} \longrightarrow \sigma_{0}, \quad s_{j, j+1} \longrightarrow \sigma_{j} \quad(j=2, \ldots, 6) .
$$

In the sequel, we frequently identify $g \in W\left(E_{7}\right)$ with $p_{W\left(E_{7}\right)}(g)$ and subgroups of $W\left(E_{7}\right)$ with their images under $p_{W\left(E_{7}\right)}$ for simplicity.

Remark 2. If $c$ is a central element of $W\left(E_{7}\right), p_{W\left(E_{7}\right)}(c)$ is the identity element of $W_{7}$.

We introduce seven polynomials defined as

$$
\begin{aligned}
p_{1}\left(x_{1}, x_{2}, x_{3}, y_{1}, y_{2}, y_{3}\right)= & \left(1-y_{2}\right)\left(x_{1}-x_{2}\right)\left(x_{3}-x_{1}+y_{1}-y_{3}+x_{1} y_{3}-x_{3} y_{1}\right) \\
& -\left(1-y_{3}\right)\left(x_{1}-x_{3}\right) \\
& \times\left(x_{2}-x_{1}+y_{1}-y_{2}+x_{1} y_{2}-x_{2} y_{1}\right), \\
p_{2}\left(x_{1}, x_{2}, x_{3}, y_{1}, y_{2}, y_{3}\right)= & x_{3}\left(x_{2}-y_{2}\right)\left(x_{1}-x_{2}\right)\left(x_{3}-x_{1}+y_{1}-y_{3}+x_{1} y_{3}-x_{3} y_{1}\right) \\
& -x_{2}\left(x_{3}-y_{3}\right)\left(x_{1}-x_{3}\right) \\
& \times\left(x_{2}-x_{1}+y_{1}-y_{2}+x_{1} y_{2}-x_{2} y_{1}\right), \\
p_{3}\left(x_{1}, x_{2}, x_{3}, y_{1}, y_{2}, y_{3}\right)= & y_{3}\left(x_{2}-y_{2}\right)\left(y_{1}-y_{2}\right)\left(x_{3}-x_{1}+y_{1}-y_{3}+x_{1} y_{3}-x_{3} y_{1}\right) \\
& -y_{2}\left(x_{3}-y_{3}\right)\left(y_{1}-y_{3}\right) \\
& \times\left(x_{2}-x_{1}+y_{1}-y_{2}+x_{1} y_{2}-x_{2} y_{1}\right), \\
p_{4}\left(x_{1}, x_{2}, x_{3}, y_{1}, y_{2}, y_{3}\right)= & x_{2} y_{3}\left(x_{1}-x_{3}\right)\left(y_{1}-y_{2}\right)-x_{3} y_{2}\left(x_{1}-x_{2}\right)\left(y_{1}-y_{3}\right), \\
p_{5}\left(x_{1}, x_{2}, x_{3}, y_{1}, y_{2}, y_{3}\right)= & x_{2} y_{3}\left(1-x_{3}\right)\left(1-y_{2}\right)-x_{3} y_{2}\left(1-x_{2}\right)\left(1-y_{3}\right), \\
p_{6}\left(x_{1}, x_{2}, x_{3}, y_{1}, y_{2}, y_{3}\right)= & x_{1} y_{3}\left(1-x_{3}\right)\left(1-y_{1}\right)-x_{3} y_{1}\left(1-x_{1}\right)\left(1-y_{3}\right), \\
p_{7}\left(x_{1}, x_{2}, x_{3}, y_{1}, y_{2}, y_{3}\right)= & x_{1} y_{2}\left(1-x_{2}\right)\left(1-y_{1}\right)-x_{2} y_{1}\left(1-x_{1}\right)\left(1-y_{2}\right) .
\end{aligned}
$$

Note that

$$
p_{4}\left(x_{1}, x_{2}, x_{3}, y_{1}, y_{2}, y_{3}\right)=x_{1} x_{2} x_{3} y_{1} y_{2} y_{3} \varphi_{1}\left(\frac{1}{x_{1}}, \frac{1}{x_{2}}, \frac{1}{x_{3}}, \frac{1}{y_{1}}, \frac{1}{y_{2}}, \frac{1}{y_{3}}\right) .
$$

Let $Q_{j}$ be the hypersurface in $\mathbf{C}^{6}$ defined by $p_{j}=0(j=1, \ldots, 7)$. Then it is easy to see that $S_{7}$ acts on the set $\left\{Q_{1}, \ldots, Q_{7}\right\}$ as permutations. We put

$$
\mathbf{P}_{0}(2,7)=\mathbf{P}(2,7)-\cup_{j=1}^{7} Q_{j}=\mathbf{C}^{6}-S_{E_{7}},
$$

where

$$
S_{E_{7}}=S_{0}\left(A_{6}\right) \cup \cup_{j=1}^{7} Q_{j} .
$$

All the elements of $W\left(E_{7}\right)$ act biregularly on $\mathbf{P}_{0}(2,7)$. 
For any subroot system $\Delta_{1}$ of type $D_{4}$ in $\Delta$, we defined in [14] a $D_{4}$-cross ratio map of the Zariski open subset $Z(\Delta)$ of the projective space $\mathbf{P}^{6}=\mathbf{P}\left(E_{\mathbf{C}}\right)$ associated to the complexification $E_{\mathbf{C}}$ of $E$ to $C R(\mathbf{P}) \simeq \mathbf{P}^{1}$. There are in total 315 subroot systems of type $D_{4}$ in $\Delta$. The corresponding $D_{4}$-cross ratio maps are denoted by $c r_{\left[i_{3}, i_{6} i_{7}\right]}^{1}(t), c r_{\left[i_{1} i_{2}, i_{3} i_{4}, i_{5} i_{6}\right]}^{2}(t), c r_{\left[i_{1} i_{2}, i_{3} i_{4}\right]}^{3}(t)(\mathrm{cf} .[14, \S 4])$. In particular,

$$
\begin{aligned}
& \operatorname{cr}_{\left[i_{1} i_{2}, i_{3} i_{4}, i_{5} i_{6}\right]}^{2}(t) \\
& \quad=\left(h_{i_{1} i_{3} i_{5}} h_{i_{2} i_{4} i_{5}} h_{i_{2} i_{3} i_{6}} h_{i_{1} i_{4} i_{6}}:-h_{i_{2} i_{3} i_{5}} h_{i_{1} i_{4} i_{5}} h_{i_{1} i_{3} i_{6}} h_{i_{2} i_{4} i_{6}}: h_{i_{1} i_{2}} h_{i_{3} i_{4}} h_{i_{5} i_{6}} h_{i_{7}}\right) .
\end{aligned}
$$

By taking the product of all 315 maps above, we obtain a map $c r_{\Delta, D_{4}}$ of $Z(\Delta)$ to $C R(\mathbf{P})^{315}$. Let $C_{E_{7}}^{\prime}$ be the image $c r_{\Delta, D_{4}}(Z(\Delta))$ and let $C_{E_{7}}$ be its closure in $C R(\mathbf{P})^{315}$.

Remark 3. In [14], we introduced three kinds of cross ratio varieties for $\Delta=\Delta\left(E_{7}\right)$ : $C\left(\Delta, A_{3}\right), C\left(\Delta, D_{4}\right), C\left(\Delta,\left\{A_{3}, D_{4}\right\}\right)$. Since we treat only $C\left(\Delta, D_{4}\right)$ in this article, we denote it by $C_{E_{7}}$ for simplicity.

We redefine the rational map $F_{E_{7}}: Z(\Delta) \longrightarrow \mathbf{P}(2,7)$ in [14, Lemma 4.2] by

$$
F_{E_{7}}(t)=\left(x_{1}(t), x_{2}(t), x_{3}(t), y_{1}(t), y_{2}(t), y_{3}(t)\right),
$$

where

$$
\begin{cases}x_{1}(t)=\frac{h_{24} \cdot h_{234} \cdot h_{15} \cdot h_{135}}{h_{14} \cdot h_{134} \cdot h_{25} \cdot h_{235}}, & y_{1}(t)=\frac{h_{34} \cdot h_{234} \cdot h_{15} \cdot h_{125}}{h_{14} \cdot h_{124} \cdot h_{35} \cdot h_{235}}, \\ x_{2}(t)=\frac{h_{24} \cdot h_{234} \cdot h_{16} \cdot h_{136}}{h_{14} \cdot h_{134} \cdot h_{26} \cdot h_{236}}, & y_{2}(t)=\frac{h_{34} \cdot h_{234} \cdot h_{16} \cdot h_{126}}{h_{14} \cdot h_{124} \cdot h_{36} \cdot h_{236}}, \\ x_{3}(t)=\frac{h_{24} \cdot h_{234} \cdot h_{17} \cdot h_{137}}{h_{14} \cdot h_{134} \cdot h_{27} \cdot h_{237}}, & y_{3}(t)=\frac{h_{34} \cdot h_{234} \cdot h_{17} \cdot h_{127}}{h_{14} \cdot h_{124} \cdot h_{37} \cdot h_{237}} .\end{cases}
$$

We identify $\mathbf{P}_{0}(2,7)$ with $C_{E_{7}}^{\prime}$ under $c r_{\Delta, D_{4}} \circ F_{E_{7}}^{-1}$.

For any subroot system $\Delta_{1}$ of $\Delta$, we defined a subvariety $Y_{\Delta, D_{4}}\left(\Delta_{1}\right)$ of $C_{E_{7}}$ in [14, §4]. A motivation for introducing such subvarieties is to find all the irreducible components of the complement of $\mathbf{P}_{0}(2,7)$ in $C_{E_{7}}$. There are four kinds of hypersurfaces of $C_{E_{7}}$ represented by $Y_{\Delta, D_{4}}\left(\Delta_{1}\right)$ for suitable subroot systems $\Delta_{1}$ which are candidates of such irreducible components. We define such hypersurfaces in a manner so that the $S_{7}$-action on them can be easily seen.

(H.1) Hypersurfaces of the 1st kind. These hypersurfaces are in a one-to-one correspondence with $\Delta^{+}$. For a root $\gamma \in \Delta$, let $\Delta_{\gamma}=\{\gamma,-\gamma\}$ be the subroot system of type $A_{1}$ in $\Delta$. We define 63 hypersurfaces of the 1st kind:

$$
Y_{i}=Y_{\Delta, D_{4}}\left(\Delta_{\gamma_{i}}\right), \quad Y_{i j}=Y_{\Delta, D_{4}}\left(\Delta_{\gamma_{i j}}\right), \quad Y_{i j k}=Y_{\Delta, D_{4}}\left(\Delta_{\gamma_{i j k}}\right) .
$$

In the sequel, we frequently write $Y_{\gamma}$ instead of $Y_{\Delta, D_{4}}\left(\Delta_{\gamma}\right)(\gamma \in \Delta)$ for simplicity. 
(H.2) Hypersurfaces of the 2nd kind. These hypersurfaces are in a one-to-one correspondence with subroot systems of type $A_{2}$ in $\Delta$. We define subroot systems of type $A_{2}$ in $\Delta$ as follows:

$$
\begin{aligned}
\Delta_{I I}\left(i_{1} i_{2} i_{3}\right) & =\left\{ \pm \gamma_{i_{1} i_{2}}, \pm \gamma_{i_{1} i_{3}}, \pm \gamma_{i_{2} i_{3}}\right\} \\
\Delta_{I I}\left(i_{1} i_{7}\right) & =\left\{ \pm \gamma_{i_{1}}, \pm \gamma_{i_{7}}, \pm \gamma_{i_{1} i_{7}}\right\} \\
\Delta_{I I}\left(i_{1} i_{2} i_{3}, i_{4} i_{5} i_{6}\right) & =\left\{ \pm \gamma_{i_{7}}, \pm \gamma_{i_{1} i_{2} i_{3}}, \pm \gamma_{i_{4} i_{5} i_{6}}\right\} \\
\Delta_{I I}\left(i_{3} i_{4}, i_{5} i_{6} i_{7}\right) & =\left\{ \pm \gamma_{i_{3} i_{4}}, \pm \gamma_{i_{1} i_{2} i_{3}}, \pm \gamma_{i_{1} i_{2} i_{4}}\right\} .
\end{aligned}
$$

Using this notation, we define 336 hypersurfaces of the 2 nd kind:

$$
\begin{array}{cl}
Z_{i_{1} i_{2} i_{3}}=Y_{\Delta, D_{4}}\left(\Delta_{I I}\left(i_{1} i_{2} i_{3}\right)\right), & Z_{i_{1} i_{7}}=Y_{\Delta, D_{4}}\left(\Delta_{I I}\left(i_{1} i_{7}\right)\right), \\
Z_{i_{1} i_{2} i_{3}, i_{4} i_{5} i_{6}}=Y_{\Delta, D_{4}}\left(\Delta_{I I}\left(i_{1} i_{2} i_{3}, i_{4} i_{5} i_{6}\right)\right), & Z_{i_{3} i_{4}, i_{5} i_{6} i_{7}}=Y_{\Delta, D_{4}}\left(\Delta_{I I}\left(i_{3} i_{4}, i_{5} i_{6} i_{7}\right)\right) .
\end{array}
$$

They are transitive under $W\left(E_{7}\right)$-action and are isomorphic to $\mathbf{T}_{4} \times \mathbf{T}_{1}$.

(H.3) Hypersurfaces of the 3rd kind. These hypersurfaces are in a one-to-one correspondence with subroot systems of type $A_{3}+A_{3}$ in $\Delta$. The following subroot systems of type $A_{3}$ in $\Delta$ exhaust these:

$$
\begin{aligned}
& \Delta_{I I I}\left(i_{1} i_{2} i_{3}\right)=\left\{ \pm \gamma_{i_{1}}, \pm \gamma_{i_{1} i_{2}}, \pm \gamma_{i_{2} i_{3}}, \pm \gamma_{i_{2}}, \pm \gamma_{i_{1} i_{3}}, \pm \gamma_{i_{3}}\right\} \\
& \Delta_{I I I}\left(i_{1} i_{2} i_{3}\right)^{\prime}=\left\{ \pm \gamma_{i_{4} i_{5}}, \pm \gamma_{i_{5} i_{6}}, \pm \gamma_{i_{6} i_{7}}, \pm \gamma_{i_{4} i_{6}}, \pm \gamma_{i_{5} i_{7}}, \pm \gamma_{i_{4} i_{7}}\right\} \\
& \Delta_{I I I}\left(i_{1} i_{3}, i_{5} i_{6} i_{7}\right)=\left\{ \pm \gamma_{i_{2}}, \pm \gamma_{i_{2} i_{4}}, \pm \gamma_{i_{1} i_{2} i_{3}}, \pm \gamma_{i_{4}}, \pm \gamma_{i_{1} i_{3} i_{4}}, \pm \gamma_{i_{5} i_{6} i_{7}}\right\}, \\
& \Delta_{I I I}\left(i_{1} i_{3}, i_{5} i_{6} i_{7}\right)^{\prime}=\left\{ \pm \gamma_{i_{2} i_{4} i_{5}}, \pm \gamma_{i_{5} i_{6}}, \pm \gamma_{i_{6} i_{7}}, \pm \gamma_{i_{2} i_{4} i_{6}}, \pm \gamma_{i_{5} i_{7}}, \pm \gamma_{i_{2} i_{4} i_{7}}\right\}, \\
& \Delta_{I I I}\left(i_{2}, i_{1} i_{5}, i_{6} i_{7}\right)=\left\{ \pm \gamma_{i_{2}}, \pm \gamma_{i_{1} i_{3} i_{5}}, \pm \gamma_{i_{3} i_{4}}, \pm \gamma_{i_{4} i_{6} i_{7}}, \pm \gamma_{i_{1} i_{4} i_{5}}, \pm \gamma_{i_{3} i_{6} i_{7}}\right\}, \\
& \Delta_{I I I}\left(i_{2}, i_{1} i_{5}, i_{6} i_{7}\right)^{\prime}=\left\{ \pm \gamma_{i_{1} i_{5}}, \pm \gamma_{i_{1} i_{2} i_{6}}, \pm \gamma_{i_{6} i_{7}}, \pm \gamma_{i_{2} i_{5} i_{6}}, \pm \gamma_{i_{1} i_{2} i_{7}}, \pm \gamma_{i_{2} i_{5} i_{7}}\right\}, \\
& \Delta_{I I I}\left(i_{1} i_{2} i_{3}, i_{5} i_{6} i_{7}\right)=\left\{ \pm \gamma_{i_{1} i_{2}}, \pm \gamma_{i_{2} i_{3}}, \pm \gamma_{i_{1} i_{2} i_{4}}, \pm \gamma_{i_{1} i_{3}}, \pm \gamma_{i_{1} i_{3} i_{4}}, \pm \gamma_{i_{2} i_{3} i_{4}}\right\} .
\end{aligned}
$$

It is clear from the definition that

$$
\begin{aligned}
\left\langle\Delta_{I I I}\left(i_{1} i_{2} i_{3}\right), \Delta_{I I I}\left(i_{1} i_{2} i_{3}\right)^{\prime}\right\rangle & =0, \\
\left\langle\Delta_{I I I}\left(i_{1} i_{3}, i_{5} i_{6} i_{7}\right), \Delta_{I I I}\left(i_{1} i_{3}, i_{5} i_{6} i_{7}\right)^{\prime}\right\rangle & =0, \\
\left\langle\Delta_{I I I}\left(i_{2}, i_{1} i_{5}, i_{6} i_{7}\right), \Delta_{I I I}\left(i_{2}, i_{1} i_{5}, i_{6} i_{7}\right)^{\prime}\right\rangle & =0, \\
\left\langle\Delta_{I I I}\left(i_{1} i_{2} i_{3}, i_{5} i_{6} i_{7}\right), \Delta_{I I I}\left(i_{5} i_{6} i_{7}, i_{1} i_{2} i_{3}\right)\right\rangle & =0 .
\end{aligned}
$$

Moreover, if $\Delta_{1}$ and $\Delta_{2}$ are subroot systems of type $A_{3}$ such that $\left\langle\Delta_{1}, \Delta_{2}\right\rangle=0$, then 
$Y_{\Delta, D_{4}}\left(\Delta_{1}\right)=Y_{\Delta, D_{4}}\left(\Delta_{2}\right)(\mathrm{cf} .[14, \S 4])$. Now we put

$$
\begin{aligned}
W_{i_{1} i_{2} i_{3}} & =Y_{\Delta, D_{4}}\left(\Delta_{I I I}\left(i_{1} i_{2} i_{3}\right)\right)\left(=Y_{\Delta, D_{4}}\left(\Delta_{I I I}\left(i_{1} i_{2} i_{3}\right)^{\prime}\right)\right), \\
W_{i_{1} i_{3}, i_{5} i_{6} i_{7}} & =Y_{\Delta, D_{4}}\left(\Delta_{I I I}\left(i_{1} i_{3}, i_{5} i_{6} i_{7}\right)\right)\left(=Y_{\Delta, D_{4}}\left(\Delta_{I I I}\left(i_{1} i_{3}, i_{5} i_{6} i_{7}\right)^{\prime}\right)\right), \\
W_{i_{2}, i_{1} i_{5}, i_{6} i_{7}} & =Y_{\Delta, D_{4}}\left(\Delta_{I I I}\left(i_{2}, i_{1} i_{5}, i_{6} i_{7}\right)\right)\left(=Y_{\Delta, D_{4}}\left(\Delta_{I I I}\left(i_{2}, i_{1} i_{5}, i_{6} i_{7}\right)^{\prime}\right)\right), \\
W_{i_{1} i_{2} i_{3}, i_{5} i_{6} i_{7}} & =Y_{\Delta, D_{4}}\left(\Delta_{I I I}\left(i_{1} i_{2} i_{3}, i_{5} i_{6} i_{7}\right)\right)\left(=Y_{\Delta, D_{4}}\left(\Delta_{I I I}\left(i_{5} i_{6} i_{7}, i_{1} i_{2} i_{3}\right)^{\prime}\right)\right),
\end{aligned}
$$

which form hypersurfaces of the $3 \mathrm{rd}$ kind. There are in total 630 hypersurfaces of the 3rd kind and they are isomorphic to $\mathbf{T}_{2} \times \mathbf{T}_{2} \times \mathbf{T}_{1}$.

(H.4) Hypersurfaces of the 4th kind. These hypersurfaces are in a one-to-one correspondence with subroot systems of type $A_{7}$ in $\Delta$. To explain the correspondence precisely, we name subroot systems of type $A_{7}$ as

$$
\begin{aligned}
\Delta_{I V}(0) & =\Delta \cap\left\langle\gamma_{1}, \gamma_{12}, \gamma_{23}, \gamma_{34}, \gamma_{45}, \gamma_{56}, \gamma_{67}\right\rangle, \\
\Delta_{I V}\left(i_{3} i_{4} i_{5}\right) & =\Delta \cap\left\langle\gamma_{i_{1} i_{2}}, \gamma_{i_{2} i_{4} i_{5}}, \gamma_{i_{3} i_{4}}, \gamma_{i_{4} i_{5}}, \gamma_{i_{3} i_{4} i_{6}}, \gamma_{i_{6} i_{7}}, \gamma_{i_{1} i_{2} i_{6}}\right\rangle .
\end{aligned}
$$

Put

$$
X_{0}=Y_{\Delta, D_{4}}\left(\Delta_{1}\right)
$$

for a subsroot system $\Delta_{1}$ of type $A_{6}$ contained in $\Delta_{I V}(0)$ and put

$$
X_{i_{3} i_{4} i_{5}}=Y_{\Delta, D_{4}}\left(\Delta_{1}\right)
$$

for a subsroot system $\Delta_{1}$ of type $A_{6}$ contained in $\Delta_{I V}\left(i_{3} i_{4} i_{5}\right)$. They are independent of the choice of $\Delta_{1}$. By virtue of [14, Lemma 4.11], we conclude that any hypersurface of the 4th kind coincides with one of $X_{0}, X_{i_{3} i_{4} i_{5}}$. There are in total 36 hypersurfaces of the 4th kind. They are transitive by $W\left(E_{7}\right)$-action and are isomorphic to $\mathbf{T}_{5}$.

Theorem 4.7 in [14] gives a description of the intersection relations among hypersurfaces of the 1st-3rd kinds in terms of root systems. Modifying this result, we obtain the intersection relations between hypersurfaces of the 4th kind and other hypersurfaces.

THEOREM 1.

(i) Two distinct hypersurfaces of the 4th kind do not intersect.

(ii) Let $\Delta_{1}$ and $\Delta_{2}$ be subroot systems of $\Delta$. Assume that $\Delta_{1}$ is of type $A_{6}$ and $\Delta_{1}^{\prime}$ is of type $A_{7}$ containing $\Delta_{1}$.

If the type of $\Delta_{2}$ is one of $A_{1}, A_{2}, A_{3}+A_{3}$, then $Y_{\Delta, D_{4}}\left(\Delta_{1}\right) \cap Y_{\Delta, D_{4}}\left(\Delta_{2}\right) \neq \emptyset$ if and only if $\Delta_{2}$ is contained in $\Delta_{1}^{\prime}$.

(iii) Let $Y$ be of the 4 th kind and let $Y^{\prime}$ be a hypersurface of $C_{E_{7}}$ such that $Y \cap Y^{\prime} \neq \emptyset$. 
If $Y^{\prime}$ is of the 1st, 2nd or 3rd kind, then $Y \cap Y^{\prime}$ is isomorphic to $\mathbf{T}_{4}, \mathbf{T}_{3} \times \mathbf{T}_{1}$ or $\mathbf{T}_{2} \times \mathbf{T}_{2}$, respectively.

Proof. Since hypersurfaces of the 4th kind are $W\left(E_{7}\right)$-transitive, we may take $Y=$ $X_{0}$. In this case, $\Delta_{1}^{\prime}=\Delta_{I V}(0)$. It is clear from the definition that $\Delta_{I V}(0)$ contains

$$
\left\{ \pm \gamma_{i}\right\}, \quad\left\{ \pm \gamma_{i j}\right\}, \quad \Delta_{I I}(i j k), \quad \Delta_{I I}(i j), \quad \Delta_{I I I}(i j k),
$$

but does not contain

$$
\begin{array}{cc}
\left\{ \pm \gamma_{i j k}\right\}, \quad \Delta_{I I}(i j k, l m n), \quad \Delta_{I I}(i j, k l m), \quad \Delta_{I I I}(i j, k l m), \\
\Delta_{I I I}(i, j k, l m), \quad \Delta_{I I I}(i j k, l m n) .
\end{array}
$$

We denote by $\operatorname{pr}_{[16,24,35]}^{2}$ the projection of $C R(\mathbf{P})^{315}$ to its factor $C R(\mathbf{P})$ corresponding to the cross ratio map $c r_{[16,24,35]}^{2}$ (cf. (4)). Then it is easy to see that

$$
p r_{[16,24,35]}^{2}\left|X_{0}=\{(1:-1: 0)\}, \quad p r_{[16,24,35]}^{2}\right| X_{123}=\{(0: 1:-1)\},
$$

which show that

$$
X_{0} \cap X_{123}=\emptyset .
$$

Since $X_{0}$ is invariant under the $S_{7}$-action, we have proved (i).

By an argument similar to the above, we find that

$$
\begin{gathered}
X_{0} \cap Y_{123}=\emptyset, \quad X_{0} \cap Z_{34,567}=\emptyset, \quad X_{0} \cap Z_{123,457}=\emptyset, \\
X_{0} \cap W_{12,567}=\emptyset, \quad X_{0} \cap W_{134,567}=\emptyset, \quad X_{0} \cap W_{5,12,67}=\emptyset .
\end{gathered}
$$

These imply that $X_{0}$ does not intersect with the hypersurfaces of the form

$$
Z_{i j k, l m n}, \quad Z_{i j, k l m}, \quad W_{i j, k l m}, \quad W_{i, j k, l m}, \quad W_{i j k, l m n} .
$$

The rest of (ii) and (iii) can be shown by direct computation.

\section{Subvarieties of hypersurfaces of the 1 st -4 th kind}

In this section, we study the structure of the intersections of hypersurfaces of the 1st4th kinds. Let $Y$ and $Y^{\prime}$ be such hypersurfaces. If one of them is of the $2 \mathrm{nd}$, 3rd or 4th kind, their intersection is described in terms of Terada models. On the other hand, if both are of the 1st kind, it is more complicated to describe their intersection. This case is treated in Section 3.4. 


\subsection{Hypersurface of the 4th kind}

There are three kinds of hypersurfaces of the form $\mathbf{T}_{5}(i j), \mathbf{T}_{5}(i j k), \mathbf{T}_{5}(i j k l)$ in the five-dimensional Terada model $\mathbf{T}_{5}$. Recall that $\mathbf{T}_{5}(i j) \simeq \mathbf{T}_{4}, \mathbf{T}_{5}(i j k) \simeq \mathbf{T}_{3} \times \mathbf{T}_{1}$, $\mathbf{T}_{5}(i j k l) \simeq \mathbf{T}_{2} \times \mathbf{T}_{2}$ and that if $\left\{i, j, k, l, i^{\prime}, j^{\prime}, k^{\prime}, l^{\prime}\right\}=\{1,2, \ldots, 8\}$, then $\mathbf{T}_{5}(i j k l)=$ $\mathbf{T}_{5}\left(i^{\prime} j^{\prime} k^{\prime} l^{\prime}\right)$. So we may take $l=8$ when we treat hypersurfaces of the form $\mathbf{T}_{5}(i j k l)$.

We study hypersurfaces of $C_{E_{7}}$ intersecting $X_{0}$ which is of the 4 th kind. Hypersurfaces of the 1st-3rd kinds intersecting $X_{0}$ are in a one-to-one correspondence with hypersurfaces of the form $\mathbf{T}_{5}(i j), \mathbf{T}_{5}(i j k), \mathbf{T}_{5}(i j k l)$ in $\mathbf{T}_{5}$. A concrete correspondence is given as follows:

$$
\begin{array}{lll}
Y_{i j} \longleftrightarrow \mathbf{T}_{5}(i j), & 1 \leq i<j \leq 7, \\
Y_{i} \longleftrightarrow \mathbf{T}_{5}(i 8), & 1 \leq i \leq 7, \\
Z_{i j k} \longleftrightarrow \mathbf{T}_{5}(i j k), & 1 \leq i<j<k \leq 7, \\
Z_{i j} \longleftrightarrow \mathbf{T}_{5}(i j 8), & 1 \leq i<j \leq 7, \\
W_{i j k} \longleftrightarrow \mathbf{T}_{5}(i j k 8), & 1 \leq i<j<k \leq 7 .
\end{array}
$$

Clearly this correspondence is $S_{7}$-equivariant.

\subsection{Hypersurface of the 2 nd kind}

Let $Y=Z_{26,345}$ be a hypersurface of the 2 nd kind and isomorphic to $\mathbf{T}_{4} \times \mathbf{T}_{1}$. Let $\pi_{1}, \pi_{2}$ be the projections of $\mathbf{T}_{4} \times \mathbf{T}_{1}$ to the first factor and second factor, respectively. If $H$ is one of $Y_{127}, Y_{167}, Y_{26}$, then $\pi_{1}(Y \cap H)=\mathbf{T}_{4}$ and $\pi_{2}(Y \cap H)$ is a point. On the other hand, if $H$ is one of the hypersurfaces of the 1st-4th kinds intersecting $Y$, then $\pi_{1}(Y \cap H)$ is a hypersurface of $\mathbf{T}_{4}$ and $\pi_{2}(Y \cap H)=\mathbf{T}_{1}$. Noting this, we find that hypersurfaces $\mathbf{T}_{4}(i j), \mathbf{T}_{4}(i j k)$ of $\mathbf{T}_{4}$ are in a one-to-one correspondence with hypersurfaces in $C_{E_{7}}$ of the 1 st -4 th kinds intersecting $Y$ and different from $Y_{127}, Y_{167}$, $Y_{26}$. A concrete correspondence is given in Table 1 .

\subsection{Hypersurface of the 3rd kind}

We take $W_{5,17,34}$ as a hypersurface of the 3rd kind and determine hypersurfaces intersecting it. By virtue of [14, Theorem 4.7] and Theorem 1, we find that the following hypersurfaces intersect $W_{5,17,34}$ :

(W.1) $Y_{234}, Z_{26,345}, Y_{346}, Y_{127}, Z_{26,157}, Y_{167}, Z_{127,346}, Z_{167,234}, Y_{26}, Y_{5}$;

(W.2) $Y_{34}, Z_{34,267}, Y_{135}, Z_{17,246}, Y_{17}, Y_{145}, Y_{357}, Y_{457}, Z_{17,236}, Z_{34,126}$;

(W.3) $X_{157}, X_{345}, Y_{256}$. 
TABLE 1.

\begin{tabular}{ll|ll|ll|ll}
\hline $\mathbf{T}_{4}(12)$ & $Y_{145}$ & $\mathbf{T}_{4}(13)$ & $X_{126}$ & $\mathbf{T}_{4}(14)$ & $Y_{135}$ & $\mathbf{T}_{4}(15)$ & $Y_{134}$ \\
$\mathbf{T}_{4}(16)$ & $Y_{7}$ & $\mathbf{T}_{4}(17)$ & $Y_{17}$ & $\mathbf{T}_{4}(23)$ & $X_{137}$ & $\mathbf{T}_{4}(24)$ & $Y_{34}$ \\
$\mathbf{T}_{4}(25)$ & $Y_{35}$ & $\mathbf{T}_{4}(26)$ & $Y_{236}$ & $\mathbf{T}_{4}(27)$ & $Y_{457}$ & $\mathbf{T}_{4}(34)$ & $X_{147}$ \\
$\mathbf{T}_{4}(35)$ & $X_{157}$ & $\mathbf{T}_{4}(36)$ & $X_{345}$ & $\mathbf{T}_{4}(37)$ & $X_{267}$ & $\mathbf{T}_{4}(45)$ & $Y_{45}$ \\
$\mathbf{T}_{4}(46)$ & $Y_{246}$ & $\mathbf{T}_{4}(47)$ & $Y_{357}$ & $\mathbf{T}_{4}(56)$ & $Y_{256}$ & $\mathbf{T}_{4}(57)$ & $Y_{347}$ \\
$\mathbf{T}_{4}(67)$ & $Y_{1}$ & & & & & & \\
$\mathbf{T}_{4}(123)$ & $W_{1,26,37}$ & $\mathbf{T}_{4}(124)$ & $Z_{34,267}$ & $\mathbf{T}_{4}(125)$ & $Z_{35,267}$ & $\mathbf{T}_{4}(126)$ & $Z_{145,236}$ \\
$\mathbf{T}_{4}(127)$ & $Z_{17,236}$ & $\mathbf{T}_{4}(134)$ & $W_{1,26,47}$ & $\mathbf{T}_{4}(135)$ & $W_{1,26,57}$ & $\mathbf{T}_{4}(136)$ & $W_{126,345}$ \\
$\mathbf{T}_{4}(137)$ & $W_{17,345}$ & $\mathbf{T}_{4}(145)$ & $Z_{45,267}$ & $\mathbf{T}_{4}(146)$ & $Z_{135,246}$ & $\mathbf{T}_{4}(147)$ & $Z_{17,246}$ \\
$\mathbf{T}_{4}(156)$ & $Z_{134,256}$ & $\mathbf{T}_{4}(157)$ & $Z_{17,256}$ & $\mathbf{T}_{4}(167)$ & $Z_{17}$ & $\mathbf{T}_{4}(234)$ & $W_{34,256}$ \\
$\mathbf{T}_{4}(235)$ & $W_{35,246}$ & $\mathbf{T}_{4}(236)$ & $W_{3,45,17}$ & $\mathbf{T}_{4}(237)$ & $W_{7,13,26}$ & $\mathbf{T}_{4}(245)$ & $Z_{345}$ \\
$\mathbf{T}_{4}(246)$ & $Z_{34,157}$ & $\mathbf{T}_{4}(247)$ & $Z_{34,126}$ & $\mathbf{T}_{4}(256)$ & $Z_{35,147}$ & $\mathbf{T}_{4}(257)$ & $Z_{35,126}$ \\
$\mathbf{T}_{4}(267)$ & $Z_{236,457}$ & $\mathbf{T}_{4}(345)$ & $W_{45,236}$ & $\mathbf{T}_{4}(346)$ & $W_{4,17,35}$ & $\mathbf{T}_{4}(347)$ & $W_{7,14,26}$ \\
$\mathbf{T}_{4}(356)$ & $W_{5,17,34}$ & $\mathbf{T}_{4}(357)$ & $W_{7,15,26}$ & $\mathbf{T}_{4}(367)$ & $W_{267,345}$ & $\mathbf{T}_{4}(456)$ & $Z_{45,137}$ \\
$\mathbf{T}_{4}(457)$ & $Z_{45,126}$ & $\mathbf{T}_{4}(467)$ & $Z_{246,357}$ & $\mathbf{T}_{4}(567)$ & $Z_{256,347}$ & & \\
\hline
\end{tabular}

Noting that $W_{5,17,34}$ is isomorphic to $\mathbf{T}_{2} \times \mathbf{T}_{2} \times \mathbf{T}_{1}$ and that there are ten lines $\mathbf{T}_{2}(i j)(1 \leq i<j \leq 5)$ in $\mathbf{T}_{2}$, we obtain a natural correspondence between the hypersurfaces in (W.1) (respectively (W.2)) and the ten lines of the first (respectively the second) factor of $\mathbf{T}_{2} \times \mathbf{T}_{2} \times \mathbf{T}_{1}$.

\subsection{Hypersurfaces of the 1st kind}

In this subsection, we always identify the root system $\Delta\left(D_{6}\right)$ of type $D_{6}$ with the subroot system of $\Delta$ whose simple roots are $\gamma_{23}, \gamma_{34}, \gamma_{123}, \gamma_{45}, \gamma_{56}, \gamma_{67}$ unless otherwise stated. Let $W\left(D_{6}\right)$ be the Weyl group of $\Delta\left(D_{6}\right)$. Then there are two $W\left(D_{6}\right)$ orbits of the set of subroot systems of type $A_{3}$ in $\Delta\left(D_{6}\right)$. We define sets of simple roots of representatives of two orbits. One is $\left\{\gamma_{23}, \gamma_{34}, \gamma_{45}\right\}$ and the other is $\left\{\gamma_{23}, \gamma_{34}, \gamma_{123}\right\}$. To distinguish these two kinds of subroot systems of type $A_{3}$, we call subroot systems belonging to the former (respectively the latter) those of type $A_{3}^{(1)}$ (respectively of type $\left.A_{3}^{(2)}\right)($ cf. $[14, \S 5])$.

If $Y$ (respectively $Z$ ) is a hypersurface of the 1 st kind (respectively the 2nd4th kind), then determining the structure of $Y \cap Z$ is reduced to determining that of Terada models. Noting this, we restrict our attention to the study of the structure of 
subvarieties in the form

$$
Y(M)=\bigcap_{\alpha \in M} Y_{\alpha}
$$

for each set $M$ consisting of mutually orthogonal positive roots of $\Delta$.

As a preparation, we classify $W\left(E_{7}\right)$-orbits of subsets of $\Delta\left(E_{7}\right)$ consisting of mutually orthogonal positive roots. The next lemma is easy to show.

LEMMA 2. If $M$ is a subset of $\Delta$ consisting of mutually orthogonal positive roots. Then, by $W\left(E_{7}\right)$-action, $M$ is transformed to one of the following sets:

$$
\begin{aligned}
M_{1} & =\left\{\gamma_{7}\right\}, \\
M_{2} & =\left\{\gamma_{7}, \gamma_{567}\right\}, \\
M_{3, A} & =\left\{\gamma_{7}, \gamma_{56}, \gamma_{567}\right\}, \quad M_{3, B}=\left\{\gamma_{7}, \gamma_{127}, \gamma_{567}\right\}, \\
M_{4, A} & =\left\{\gamma_{7}, \gamma_{34}, \gamma_{127}, \gamma_{567}\right\}, \quad M_{4, B}=\left\{\gamma_{7}, \gamma_{34}, \gamma_{56}, \gamma_{567}\right\}, \\
M_{5} & =\left\{\gamma_{7}, \gamma_{34}, \gamma_{56}, \gamma_{127}, \gamma_{567}\right\}, \\
M_{6} & =\left\{\gamma_{7}, \gamma_{12}, \gamma_{34}, \gamma_{56}, \gamma_{127}, \gamma_{567}\right\}, \\
M_{7} & =\left\{\gamma_{7}, \gamma_{12}, \gamma_{34}, \gamma_{56}, \gamma_{127}, \gamma_{347}, \gamma_{567}\right\} .
\end{aligned}
$$

Remark 4. Lemma 2 is related to the classification of conjugacy classes of Cartan subalgebras of a normal real form $\mathfrak{g}$ of a simple Lie algebra of type $E_{7}$. Indeed, there is a natural one-to-one correspondence between the set $\left\{M_{j}(j=1,2,5,6,7)\right.$, $\left.M_{j, C}(j=3,4, C=A, B), \emptyset\right\}$ and that of conjugacy classes of Cartan subalgebras of $\mathfrak{g}$.

Let $\mathbf{M}_{j}(j=1,2,5,6,7)$ (respectively $\left.\mathbf{M}_{j, A}, \mathbf{M}_{j, B}(j=3,4)\right)$ be the $W\left(E_{7}\right)$ orbit of $M_{j}(j=1,2,5,6,7)$ (respectively $\left.M_{j, A}, M_{j, B}(j=3,4)\right)$. The lemma below distinguishes $\mathbf{M}_{j, A}$ and $\mathbf{M}_{j, B}(j=3,4)$. Its proof is straightforward.

LEMMA 3.

(i) $\quad M_{3, A}^{\perp}$ is a root system of type $D_{4}$ and $M_{3, B}^{\perp}$ is a root system of type $3 A_{1}$.

(ii) $M_{4, A}^{\perp} \cap \Delta^{+} \in \mathbf{M}_{3, A}, M_{4, B}^{\perp} \cap \Delta^{+} \in \mathbf{M}_{3, B}$.

We study subvarieties $Y\left(M_{j}\right)(j=1,2,5,6,7)$ and $Y\left(M_{j, C}\right)(j=3,4, C=$ $A, B)$, separately.

( $Y .1)$ The case $Y\left(M_{1}\right)$. From the definition, $Y\left(M_{1}\right)=Y_{7}$. We collect hypersurfaces intersecting $Y_{7}$ in Table 2. We use the indices $i, j, k, l, n, m$ as distinct numbers among $1, \ldots, 6$. (In Table 2, we use the notation $R(Y) ; R(Y)$ means a subroot system of $\Delta$ such that $Y=Y_{\Delta, D_{4}}(R(Y))$. Though $R(Y)$ is not uniquely determined by $Y$, for 
TABLE 2.

\begin{tabular}{lllll}
\hline Kinds & $Y$ & $Y \cap Y_{7}$ & $R(Y) \cap R\left(Y_{7}\right)^{\perp}$ & {$[\mathbf{1 4}, \S 5]$} \\
\hline \multirow{2}{*}{ 1st kind } & $Y_{i j}, Y_{i j 7}$ & $C\left(\Delta\left(D_{5}\right),\left\{A_{3}^{(1)}, D_{4}\right\}\right)$ & $D_{6}$-root & $(5.5 .1)$ \\
2nd kind & $Z_{i 7}, Z_{i j k, l m n}$ & $\mathbf{T}_{4}$ & $A_{5}$ & $(5.5 .7)$ \\
& $Z_{i j, k l m}, Z_{i j k}$ & $\mathbf{T}_{3} \times \mathbf{T}_{1}$ & $A_{3}^{(2)}+A_{2}$ & $(5.5 .2)$ \\
\multirow{4}{*}{ 3rd kind } & $W_{i j 7}, W_{i j, k l m}, W_{7, i j, k l}$ & $\mathbf{T}_{2} \times \mathbf{T}_{1} \times \mathbf{T}_{1}$ & $A_{3}^{(1)}+A_{1}+A_{1}$ & $(5.5 .3)$ \\
& $W_{i j k, l m n}$ & $\mathbf{T}_{2} \times \mathbf{T}_{2}$ & $A_{3}^{(2)}+A_{3}^{(2)}$ & $(5.5 .4)$ \\
4th kind & $X_{0}, X_{i j 7}$ & $\mathbf{T}_{4}$ & $A_{5}$ & $(5.5 .7)$ \\
\hline
\end{tabular}

our purpose, it is sufficient to choose a suitable one. In particular, we have taken $R\left(Y_{7}\right)=\left\{ \pm \gamma_{7}\right\}$.)

(Y.2) The case $Y\left(M_{2}\right)$. Noting that $Y\left(M_{2}\right)=Y_{7} \cap Y_{567}$, we determine hypersurfaces intersecting both $Y_{7}$ and $Y_{567}$. For the moment, let $\mathbf{R}^{6}$ be a six-dimensional inner product space with an orthonormal basis $\left\{\mathbf{e}_{j} ; j=1, \ldots, 6\right\}$. The root system $\Delta\left(D_{6}\right)$ of type $D_{6}$ is identified with the set

$$
\left\{\mathbf{e}_{i} \pm \mathbf{e}_{j} ; i, j=1, \ldots, 6, i \neq j\right\} .
$$

In the sequel, we regard $\Delta\left(D_{6}\right)$ as a subroot system of $\Delta$ by

$$
\mathbf{e}_{i}-\mathbf{e}_{j} \longrightarrow \gamma_{i j}, \quad \mathbf{e}_{i}+\mathbf{e}_{j} \longrightarrow \gamma_{i j 7}
$$

For a subroot system of type $A_{3}^{(1)}$ (respectively $D_{4}$ ), we obtain an $A_{3}$-cross ratio map $\operatorname{cr}_{A_{3}, \Delta_{1}}$ (respectively a $D_{4}$-cross ratio map $\left.\operatorname{cr}_{D_{4}, \Delta_{1}}\right)$ of $Z\left(\Delta\left(D_{6}\right)\right)$ to $C R(\mathbf{P})$, where $Z\left(\Delta\left(D_{6}\right)\right)$ is the complement of root hyperplanes of $\mathbf{P}^{5}$. The maps

$$
\begin{aligned}
& t \longrightarrow\left(\left(t_{1}-t_{2}\right)\left(t_{3}-t_{4}\right):-\left(t_{1}-t_{3}\right)\left(t_{2}-t_{4}\right):\left(t_{1}-t_{4}\right)\left(t_{2}-t_{3}\right)\right) \\
& t \longrightarrow\left(\left(t_{1}^{2}-t_{2}^{2}\right)\left(t_{3}^{2}-t_{4}^{2}\right):-\left(t_{1}^{2}-t_{3}^{2}\right)\left(t_{2}^{2}-t_{4}^{2}\right):\left(t_{1}^{2}-t_{4}^{2}\right)\left(t_{2}^{2}-t_{3}^{2}\right)\right)
\end{aligned}
$$

for $t=\sum_{i=1}^{6} t_{i} \mathbf{e}_{i}$ are examples of $A_{3}$-cross ratio maps and $D_{4}$-cross ratio maps. By using both $A_{3}$-cross ratio maps and $D_{4}$-cross ratio maps $W\left(D_{6}\right)$-transitive to (8) or (9), we defined the varicty $C\left(\Delta\left(D_{6}\right),\left\{A_{3}^{(1)}, D_{4}\right\}\right)$ in $[14, \S 5]$.

We consider subvarieties of $C\left(\Delta\left(D_{6}\right),\left\{A_{3}^{(1)}, D_{4}\right\}\right)$ corresponding to subroot systems of type $A_{1}$. Taking a subroot system $\Delta_{0}=\{ \pm \alpha\}$ of type $A_{1}$, where $\alpha=\mathbf{e}_{5}+$ $\mathbf{e}_{6} \in \Delta\left(D_{6}\right)$, we find from the correspondence (7) that $Y_{\Delta\left(D_{6}\right),\left\{A_{3}^{(1)}, D_{4}\right\}}\left(\Delta_{0}\right)=Y\left(M_{2}\right)$. 
LEMMA 4. For the set $\Delta_{0}=\{ \pm \alpha\}$ defined above, $Y_{\Delta\left(D_{6}\right),\left\{A_{3}^{(1)}, D_{4}\right\}}\left(\Delta_{0}\right)$ is a closed subvariety of $C R(\mathbf{P})^{57}$ and there is a natural birational map of $Y_{\Delta\left(D_{6}\right),\left\{A_{3}^{(1)}, D_{4}\right\}}\left(\Delta_{0}\right)$ onto $C\left(\Delta\left(D_{5}\right),\left\{A_{3}^{(1)}, D_{4}\right\}\right)$.

Proof. Noting that $\alpha(t)=t_{5}+t_{6}$, we first determine such $A_{3}$-cross ratio (respectively $D_{4}$-cross ratio) maps $W\left(D_{6}\right)$-transitive to (8) (respectively (9)) that the closures of images of the restrictions to the hyperplane $t_{5}+t_{6}=0$ are $\mathbf{P}^{1}$. They are classified into three kinds of maps which are $G$-transitive to the maps defined in (i.A), (i.B), (ii) below, where $G$ is the subgroup of $W\left(D_{6}\right)$ leaving the hyperplane $t_{5}+t_{6}=0$ fixed.

(i.A) $A_{3}$-cross ratio maps $I$.

$$
\begin{gathered}
t \longrightarrow\left(\left(t_{i_{1}}-t_{i_{2}}\right)\left(t_{i_{3}}-t_{i_{4}}\right):-\left(t_{i_{1}}-t_{i_{3}}\right)\left(t_{i_{2}}-t_{i_{4}}\right):\left(t_{i_{1}}-t_{i_{4}}\right)\left(t_{i_{2}}-t_{i_{3}}\right)\right), \\
\left\{i_{1}, i_{2}, i_{3}, i_{4}\right\} \subset\{1,2,3,4,5\} .
\end{gathered}
$$

(i.B) $A_{3}$-cross ratio maps II.

$$
\begin{gathered}
t \longrightarrow\left(\left(t_{i_{1}}-t_{i_{2}}\right)\left(t_{5}-t_{6}\right):-\left(t_{i_{1}}-t_{5}\right)\left(t_{i_{2}}-t_{6}\right):\left(t_{i_{1}}-t_{6}\right)\left(t_{i_{2}}-t_{5}\right)\right), \\
\left\{i_{1}, i_{2}\right\} \subset\{1,2,3,4\} .
\end{gathered}
$$

(ii) $D_{4}$-cross ratio maps.

$$
\begin{gathered}
t \longrightarrow\left(\left(t_{i_{1}}^{2}-t_{i_{2}}^{2}\right)\left(t_{i_{3}}^{2}-t_{i_{4}}^{2}\right):-\left(t_{i_{1}}^{2}-t_{i_{3}}^{2}\right)\left(t_{i_{2}}^{2}-t_{i_{4}}^{2}\right):\left(t_{i_{1}}^{2}-t_{i_{4}}^{2}\right)\left(t_{i_{2}}^{2}-t_{i_{3}}^{2}\right)\right), \\
\left\{i_{1}, i_{2}, i_{3}, i_{4}\right\} \subset\{1,2,3,4,5\} .
\end{gathered}
$$

There are 40 distinct maps (respectively 12 maps, 5 maps) $G$-transitive to (10) (respectively (11), (12)). Let $\Gamma_{1}$ (respectively $\Gamma_{2}, \Gamma_{3}$ ) be the map defined as the product of such 40 maps (respectively 12 maps, 5 maps). Then $\Gamma_{1} \times \Gamma_{2} \times \Gamma_{3}$ induces an embedding $\iota$ of $Y_{\Delta\left(D_{6}\right),\left\{A_{3}^{(1)}, D_{4}\right\}}\left(\Delta_{0}\right)$ into $C R(\mathbf{P})^{57}=C R(\mathbf{P})^{40} \times C R(\mathbf{P})^{12} \times C R(\mathbf{P})^{5}$. Similarly, $\Gamma_{1} \times \Gamma_{3}$ induces an embedding of $C\left(\Delta\left(D_{5}\right),\left\{A_{3}^{(1)}, D_{4}\right\}\right)$ into $C R(\mathbf{P})^{45}=$ $C R(\mathbf{P})^{40} \times C R(\mathbf{P})^{5}$. Moreover, the composition of $\iota$ and the natural projection

$$
C R(\mathbf{P})^{40} \times C R(\mathbf{P})^{12} \times C R(\mathbf{P})^{5} \longrightarrow C R(\mathbf{P})^{40} \times C R(\mathbf{P})^{5}=C R(\mathbf{P})^{45}
$$

induces a surjection $\rho$ of $Y_{\Delta\left(D_{6}\right),\left\{A_{3}^{(1)}, D_{4}\right\}}\left(\Delta_{0}\right)$ to $C\left(\Delta\left(D_{5}\right),\left\{A_{3}^{(1)}, D_{4}\right\}\right)$. It is clear from the construction that $\rho$ is birational and onto.

We now study hypersurfaces of the form $Y_{\Delta\left(D_{6}\right),\left\{A_{3}^{(1)}, D_{4}\right\}}\left(\Delta_{1}\right)$ in $C\left(\Delta\left(D_{6}\right),\left\{A_{3}^{(1)}\right.\right.$, $\left.D_{4}\right\}$ ) corresponding to subroot systems $\Delta_{1}$ of $\Delta\left(D_{6}\right)$ (cf. [14, §5]). The types of $\Delta_{1}$ and their properties are shown in Table 3. 
TABle 3.

\begin{tabular}{llll}
\hline & Type of $\Delta_{1}$ & $Y_{\Delta\left(D_{6}\right),\left\{A_{3}^{(1)}, D_{4}\right\}}\left(\Delta_{1}\right)$ & {$[\mathbf{1 4}, \S 5]$} \\
\hline (III.1) & $A_{1}$ & Subvariety of $C R(\mathbf{P})^{57}$ & $(5.5 .1)$ \\
(III.2) & $A_{2}$ & $\mathbf{T}_{3} \times \mathbf{T}_{1}$ & $(5.5 .2)$ \\
(III.3) & $A_{3}^{(1)}$ & $\mathbf{T}_{2} \times \mathbf{T}_{1} \times \mathbf{T}_{1}$ & $(5.5 .3)$ \\
(III.4) & $A_{3}^{(2)}$ & $\mathbf{T}_{2} \times \mathbf{T}_{2}$ & $(5.5 .4)$ \\
(III.5) & $A_{5}$ & $\mathbf{T}_{4}$ & $(5.5 .7)$ \\
(III.6) & $D_{5}$ & Remark 5 & $(5.5 .8)$ \\
\hline
\end{tabular}

Remark 5. (A relation between hypersurfaces in (III.6) and configurations of seven points in $\mathbf{P}(2,7)$.) Under the identification of $\mathbf{P}(2,7)$ with the subset of the $\left(x_{1}, x_{2}, x_{3}, y_{1}, y_{2}, y_{3}\right)$-space, we find from [14, Proposition 5.4] that the hypersurface $Y_{1}$ (respectively $Y_{7}$ ) of $C_{E_{7}}$ is birational to the hypersurface $Q_{1}$ (respectively $Q_{7}$ ) of the $(x, y)$-space introduced in Section 2.

We now take $\Delta_{1}=\left\{ \pm \mathbf{e}_{i} \pm \mathbf{e}_{j} ; 1 \leq i<j \leq 5\right\}$ as a root system of type $D_{5}$ and identify $\Delta_{1}$ with a subset of $\Delta$ by (7). Then, the hypersurface $Y_{\Delta\left(D_{6}\right),\left\{A_{3}^{(1)}, D_{4}\right\}}\left(\Delta_{1}\right)$ of $Y_{7}\left(\simeq C\left(\Delta\left(D_{6}\right),\left\{A_{3}^{(1)}, D_{4}\right\}\right)\right)$ is birational to the subvariety of $\mathbf{C}^{6}$ defined by

$$
\left\{\begin{array}{l}
x_{1} x_{2} y_{1}-x_{1} x_{2} y_{2}-x_{1} y_{1} y_{2}+x_{1} y_{2}+x_{2} y_{1} y_{2}-x_{2} y_{1}=0, \\
x_{2}^{2} y_{2}-x_{2}^{2} y_{3}-x_{2} y_{2}^{2}+x_{2} y_{3}+x_{3} y_{2}^{2}-x_{3} y_{2}=0 .
\end{array}\right.
$$

The first equation of (13) is nothing but $p_{7}=0$.

We explain a geometric meaning of (13). For this purpose, we consider seven points $P_{1}, \ldots, P_{7}$ of $\mathbf{P}^{2}$ :

$$
\begin{gathered}
P_{1}=(1: 0: 0), \quad P_{2}=(0: 1: 0), \quad P_{3}=(0: 0: 1), \quad P_{4}=(1: 1: 1), \\
P_{5}=\left(1: x_{1}: y_{1}\right), \quad P_{6}=\left(1: x_{2}: y_{2}\right), \quad P_{7}=\left(1: x_{3}: y_{3}\right) .
\end{gathered}
$$

We assume that the seven points above are in general position. Then we have the claim below.

ClaIM. Equation (13) is equivalent to the condition that there is a conic $C$ of $\mathbf{P}^{2}$ satisfying (i), (ii) below:

(i) $C$ passes through the six points $P_{1}, \ldots, P_{6}$;

(ii) the point $P_{7}$ is on the line tangent to $C$ at $P_{6}$. 
TABLE 4.

\begin{tabular}{|c|c|c|c|c|c|c|c|}
\hline & (R.1) & (R.2) & (R.3) & (R.4) & (R.5) & (R.6) & (R.7) \\
\hline (IV.1) & $A_{1}$ & (R.2.a) & 12 & (R.4.1) & (R.5.1) & $\mathbf{T}_{3}$ & $Y_{12}, Y_{127}$ \\
\hline (IV.2) & $A_{1}$ & (R.2.b) & 1 & (R.4.2) & (R.5.2) & (R.6.2) & $Y_{56}$ \\
\hline (IV.3) & $A_{2}$ & (R.2.a) & 16 & $\mathbf{T}_{3} \times \mathbf{T}_{1}$ & $\mathbf{T}_{2} \times \mathbf{T}_{1}$ & $\mathbf{T}_{1} \times \mathbf{T}_{1} \times \mathbf{T}_{1}$ & $Z_{123}, Z_{23,456}$ \\
\hline (IV.4) & $A_{2}$ & (R.2.b) & 8 & $\mathbf{T}_{3} \times \mathbf{T}_{1}$ & $\mathbf{T}_{3}$ & $\mathbf{T}_{3}$ & $Z_{15,234}$ \\
\hline (IV.5) & $A_{3}^{(1)}$ & (R.2.a) & 8 & $\mathbf{T}_{2} \times \mathbf{T}_{1} \times \mathbf{T}_{1}$ & $\mathbf{T}_{2} \times \mathbf{T}_{1}$ & $\mathbf{T}_{2}$ & $\begin{array}{l}W_{56,123}, W_{567} \\
W_{7,12,34}\end{array}$ \\
\hline (IV.6) & $A_{3}^{(1)}$ & (R.2.b) & 24 & $\mathbf{T}_{2} \times \mathbf{T}_{1} \times \mathbf{T}_{1}$ & $\mathbf{T}_{2} \times \mathbf{T}_{1}$ & $\mathbf{T}_{1} \times \mathbf{T}_{1} \times \mathbf{T}_{1}$ & $W_{12,345}, W_{7,15,26}$ \\
\hline (IV.7) & $A_{3}^{(2)}$ & (R.2.a) & 4 & $\mathbf{T}_{2} \times \mathbf{T}_{2}$ & $\mathbf{T}_{2} \times \mathbf{T}_{1}$ & $\mathbf{T}_{2}$ & $W_{123,456}$ \\
\hline (IV.8) & $A_{5}$ & (R.2.b) & 16 & $\mathbf{T}_{4}$ & $\mathbf{T}_{3}$ & $\mathbf{T}_{3}$ & $X_{167}, Z_{67}, Z_{146,235}$ \\
\hline
\end{tabular}

As a consequence of the Claim above, we find that $Y_{7}$ is a compactification of the subvariety of $\mathbf{P}_{0}(2,7)$ consisting of the $P G L(3)$-orbits of $\left(P_{1}, \ldots, P_{7}\right)$ satisfying (i) and (ii) of the Claim.

The next task is to study hypersurfaces of $C_{E_{7}}$ intersecting $Y\left(M_{2}\right)\left(\simeq Y_{\gamma_{567}}^{(1)}=\right.$ $\left.Y_{\Delta\left(D_{6}\right),\left\{A_{3}^{(1)}, D_{4}\right\}}\left(\left\{ \pm \gamma_{567}\right\}\right)\right)$. Let

$$
\rho: Y_{\gamma_{567}}^{(1)} \longrightarrow C\left(\Delta\left(D_{5}\right),\left\{A_{3}^{(1)}, D_{4}\right\}\right)
$$

be the surjection given in the proof of Lemma 4 . There are eight kinds of hypersurfaces of the form $Y_{\Delta\left(D_{6}\right),\left\{A_{3}^{(1)}, D_{4}\right\}}\left(\Delta_{1}\right)$ in $C\left(\Delta\left(D_{6}\right),\left\{A_{3}^{(1)}, D_{4}\right\}\right)$ intersecting $Y_{\gamma_{567}}^{(1)}$, where $\Delta_{1}$ are subroot systems of $\Delta\left(D_{6}\right)$ which correspond to (IV.1)-(IV.8) in Table 4 below. Since $Y_{7}$ is identified with $C\left(\Delta\left(D_{6}\right),\left\{A_{3}^{(1)}, D_{4}\right\}\right)$, Table 4 also shows the hypersurfaces of $C_{E_{7}}$ intersecting $Y\left(M_{2}\right)$.

We give some remarks on how to read Table 4.

(TA4.1) The type of $\Delta_{1}$ is contained in the column (R.1).

(TA4.2) The condition (R.2.a) means that $\Delta_{1} \subset \Delta\left(D_{4}\right)$ and (R.2.b) means that $\gamma_{567} \in \Delta_{1}$.

(TA4.3) The numbers of hypersurfaces are given in column (R.3). (There are in total 89 hypersurfaces of the form $Y_{\Delta\left(D_{6}\right),\left\{A_{3}^{(1)}, D_{4}\right\}}\left(\Delta_{1}\right)$ in $C\left(\Delta\left(D_{6}\right),\left\{A_{3}^{(1)}, D_{4}\right\}\right)$ intersecting $Y_{\gamma_{567}}^{(1)}$.)

(TA4.4) The varieties isomorphic to the intersection with $C\left(\Delta\left(D_{6}\right),\left\{A_{3}^{(1)}, D_{4}\right\}\right)$ 
are given in column (R.4). In particular, the intersection of the subvariety in (IV.1) (respectively (IV.2)) and $Y_{\gamma_{567}}^{(1)}$ is isomorphic to $Y(M)=\cap_{\beta \in M} Y_{\beta}$, where $M$ is a subset of $\Delta$ consisting of two distinct positive roots (cf. Lemma 2). We shall study subvarieties of these types later in this section.

(TA4.5) In column (R.5), varieties isomorphic to the intersection of the varieties in question and $Y_{\gamma_{567}}^{(1)}$ are given. We shall study subvarieties in (R.5.1) and (R.5.2) (which are isomorphic to $Y\left(M_{3, B}\right), Y\left(M_{3, A}\right)$, respectively) later in this section.

(TA4.6) In column (R.6), subvarieties of $C\left(\Delta\left(E_{6}\right), D_{4}\right)$ isomorphic to the image by the map $\rho$ of the subvarieties in (R.5) are given. In particular, subvarieties in (IV.5) and (IV.7) are not hypersurfaces in $C\left(\Delta\left(E_{6}\right), D_{4}\right)$. We note here that $C\left(\Delta\left(E_{6}\right), D_{4}\right)$ is Naruki's cross ratio variety and that there are 76 hypersurfaces in it (cf. [9]). There is a natural one-to-one correspondence between the subvarieties in column (R.6) which are still hypersurfaces in $C\left(\Delta\left(E_{6}\right), D_{4}\right)$ except that in (IV.2) and the 76 hypersurfaces given in [9].

We now consider the subvariety in (IV.2). In this case, $\Delta_{1}=\left\{ \pm\left(\mathbf{e}_{5}-\mathbf{e}_{6}\right)\right\}$. We put

$$
Y=Y_{\gamma_{567}}^{(1)} \cap Y_{\Delta\left(D_{6}\right),\left\{A_{3}^{(1)}, D_{4}\right\}}\left(\left\{ \pm\left(\mathbf{e}_{5}-\mathbf{e}_{6}\right)\right\}\right) .
$$

Then $Y$ and its image $\rho(Y)$ by $\rho$ (cf. (15)) are the varieties in (R.5.2) and (R.6.2), respectively. It is easy to show that $Y \simeq \rho(Y)$ and that $\rho(Y)$ is identified with one of the 45 exceptional divisors of Naruki's cross ratio variety (cf. $[9,13])$.

(TA4.7) In the column (R.7), examples of hypersurfaces in $C_{E_{7}}$ intersecting $Y_{7} \cap Y_{567}$ are shown.

From the data in (R.7), we can determine all the hypersurfaces of the form $Y_{\Delta, D_{4}}\left(\Delta_{0}\right)$ in $C_{E_{7}}$ intersecting $Y_{7} \cap Y_{567}$ as follows, where $\Delta_{0}$ is a subroot system of $\Delta$. In the cases (IV.1)-(IV.7), $\Delta_{0}$ is so taken that $\Delta_{0}$ is contained in $\Delta\left(D_{6}\right)$. Then $Y_{\Delta, D_{4}}\left(\Delta_{0}\right) \cap Y_{7}$ coincides with $Y_{\Delta\left(D_{6}\right),\left\{A_{3}^{(1)}, D_{4}\right\}}\left(\Delta_{0}\right)$. On the other hand, in the case (IV.8), $Y_{\Delta, D_{4}}\left(\Delta_{0}\right) \cap Y_{7}$ coincides with $Y_{\Delta\left(D_{6}\right),\left\{A_{3}^{(1)}, D_{4}\right\}}\left(\Delta_{0} \cap \Delta\left(D_{6}\right)\right)$. For this reason, all the hypersurface of the 1st-4th kinds intersecting $Y\left(M_{2}\right)$ are obtained from those in (R.7), by permutations among the indices 1, 2, 3, 4 and the transposition of 5, 6 .

(Y.3) The cases $Y\left(M_{3, A}\right), Y\left(M_{3, B}\right)$. We treat subvarieties $Y\left(M_{3, A}\right)=\cap_{\beta \in M_{3, A}} Y_{\beta}$ and $Y\left(M_{3, B}\right)=\cap_{\beta \in M_{3, B}} Y_{\beta}$ of $C_{E_{7}}$, separately.

(Y.3.A) The case $Y\left(M_{3, A}\right)$. It follows from the definition that $Y\left(M_{3, A}\right)$ is embedded in $C R(\mathbf{P})^{29}$ as a closed subvariety. Moreover, $\rho\left(Y\left(M_{3, A}\right)\right) \simeq Y\left(M_{3, A}\right)$ and as we noted before, $Y\left(M_{3, A}\right)$ is isomorphic to one of 45 exceptional divisors in Naruki's cross ratio variety (cf. $[9,13])$. 
There are 40 hypersurfaces of $C_{E_{7}}$ intersecting $Y\left(M_{3, A}\right)$. Such hypersurfaces are given as follows:

$$
\begin{aligned}
& Y_{12}, Y_{13}, Y_{14}, Y_{23}, Y_{24}, Y_{34}, Y_{127}, Y_{137}, Y_{147}, Y_{237}, Y_{247}, Y_{347}, \\
& Z_{123}, Z_{124}, Z_{134}, Z_{234}, Z_{23,456}, Z_{24,356}, Z_{34,256}, Z_{13,456}, Z_{14,356}, Z_{34,156}, \\
& Z_{12,456}, Z_{14,256}, Z_{24,156}, Z_{12,356}, Z_{13,256}, Z_{23,156}, \\
& W_{567}, W_{56,234}, W_{56,134}, W_{56,124}, W_{56,123}, W_{7,12,34}, W_{7,13,24}, W_{7,14,23}, \\
& W_{156,234}, W_{256,134}, W_{356,124}, W_{456,123} .
\end{aligned}
$$

(Y.3.B) The case $Y\left(M_{3, B}\right)$. It is easy to show that $Y\left(M_{3, B}\right)$ is embedded in $C R(\mathbf{P})^{22}$ as a closed subvariety. Moreover, $\rho\left(Y\left(M_{3, B}\right)\right)$ is isomorphic to $\mathbf{T}_{3}$. There are in total 32 hypersurfaces of $C_{E_{7}}$ intersecting $Y\left(M_{3, B}\right)$. They are given as follows:

$$
\begin{aligned}
& Y_{12}, Y_{34}, Y_{347}, Y_{56}, X_{167}, X_{267}, X_{157}, X_{257}, \\
& Z_{135,246}, Z_{145,236}, Z_{136,245}, Z_{146,235}, \\
& Z_{23,456}, Z_{24,356}, Z_{13,456}, Z_{14,356}, Z_{35,124}, Z_{45,123}, Z_{36,124}, Z_{46,123}, \\
& W_{7,35,46}, W_{7,45,36}, W_{7,13,24}, W_{7,14,23}, W_{7,15,26}, W_{7,16,25}, W_{356,124}, W_{456,123}, \\
& W_{12,345}, W_{12,346}, W_{56,234}, W_{56,134} .
\end{aligned}
$$

(Y.4) The cases $Y\left(M_{4, A}\right), Y\left(M_{4, B}\right)$. We treat the subvarieties $Y\left(M_{4, A}\right)=\cap_{\beta \in M_{4, A}} Y_{\beta}$ and $Y\left(M_{4, B}\right)=\cap_{\beta \in M_{4, B}} Y_{\beta}$ of $C_{E_{7}}$.

We note the lemma below without proof, because we do not use it in the subsequent discussion.

LEMmA 5. The subvarieties $Y\left(M_{4, A}\right)$ and $Y\left(M_{4, B}\right)$ are isomorphic.

To determine the structure of the varieties $Y\left(M_{4, A}\right)$ and $Y\left(M_{4, B}\right)$, we introduce a subvariety $Z$ of $\mathbf{P}^{2} \times \mathbf{P}^{2}$ defined by

$$
\left\{\left(\left(\xi_{1}: \xi_{2}: \xi_{3}\right),\left(\eta_{1}: \eta_{2}: \eta_{3}\right)\right) \in \mathbf{P}^{2} \times \mathbf{P}^{2} ; \xi_{1} \eta_{1}=\xi_{2} \eta_{2}=\xi_{3} \eta_{3}\right\} .
$$

Moreover, we define 10 lines of $Z$ by

$$
\begin{array}{lll}
L_{1}: & \xi_{2}+\xi_{3}=0, & \eta_{2}+\eta_{3}=0 \\
L_{2}: \xi_{3}+\xi_{1}=0, & \eta_{3}+\eta_{1}=0 \\
L_{3}: \xi_{1}+\xi_{2}=0, & \eta_{1}+\eta_{2}=0 \\
L_{0}: & \xi_{1}+\xi_{2}+\xi_{3}=0, & \eta_{1}+\eta_{2}+\eta_{3}=0
\end{array}
$$


TABLE 5.

\begin{tabular}{llll}
\hline$\tilde{Z}$ & $Y\left(M_{4, B}\right)$ & $Y\left(M_{4, A}\right)$ & $\rho\left(M_{4, A}\right)$ \\
\hline$L_{0}$ & $Z_{34,156}$ & $X_{167}$ & $\mathbf{T}_{2}(45)$ \\
$L_{1}$ & $Y_{12}$ & $Y_{12}$ & $\mathbf{T}_{2}(35)$ \\
$L_{2}$ & $Y_{127}$ & $Y_{56}$ & $Y_{56}^{*}$ \\
$L_{3}$ & $Y_{347}$ & $Y_{347}$ & $\mathbf{T}_{2}(24)$ \\
$M_{1}$ & $W_{567}$ & $W_{12,346}$ & $\mathbf{T}_{2}(14)$ \\
$M_{2}$ & $W_{56,234}$ & $W_{56,134}$ & $\mathbf{T}_{2}(25) \cap \mathbf{T}_{2}(34) \cap Y_{56}^{*}$ \\
$M_{3}$ & $W_{134,256}$ & $W_{7,15,26}$ & $\mathbf{T}_{2}(15)$ \\
$N_{1}$ & $Z_{34,256}$ & $X_{267}$ & $\mathbf{T}_{2}(34)$ \\
$N_{2}$ & $Z_{134}$ & $X_{157}$ & $\mathbf{T}_{2}(23)$ \\
$N_{3}$ & $Z_{234}$ & $X_{257}$ & $\mathbf{T}_{2}(25)$ \\
$E_{1}$ & $W_{7,12,34}$ & $W_{12,345}$ & $\mathbf{T}_{2}(12)$ \\
$E_{2}$ & $W_{56,134}$ & $W_{56,234}$ & $\mathbf{T}_{2}(23) \cap \mathbf{T}_{2}(45) \cap Y_{56}^{*}$ \\
$E_{3}$ & $W_{156,234}$ & $W_{7,16,25}$ & $\mathbf{T}_{2}(13)$ \\
\hline
\end{tabular}

$$
\begin{array}{lll}
M_{1}: & \xi_{2}=\xi_{3}=0, & \eta_{1}=0 \\
M_{2}: & \xi_{3}=\xi_{1}=0, & \eta_{2}=0 \\
M_{3}: & \xi_{1}=\xi_{2}=0, & \eta_{3}=0 \\
N_{1}: & \eta_{2}=\eta_{3}=0, & \xi_{1}=0 \\
N_{2}: & \eta_{3}=\eta_{1}=0, & \xi_{2}=0 \\
N_{3}: & \eta_{1}=\eta_{2}=0, & \xi_{3}=0
\end{array}
$$

The three lines $L_{j}, N_{j}, L_{0}$ intersect at a point $(j=1,2,3)$. Noting this, we define the space $\tilde{Z}$ obtained from $Z$ by blowing up at these three points $L_{j} \cap N_{j} \cap L_{0}$ ( $j=1,2,3$ ) and let $\pi$ be the natural projection of $\tilde{Z}$ to $Z$. We denote by $E_{j}$ the exceptional fibre $\pi^{-1}\left(L_{j} \cap N_{j}\right)(j=1,2,3)$ and by $\tilde{L}_{0}, \tilde{L}_{i}, \tilde{M}_{i}, \tilde{N}_{i}$ proper transforms of $L_{0}, L_{i}, M_{i}, N_{i}$, respectively. In this way, we obtain 13 lines on $\tilde{Z}$.

We determine a one-to-one correspondence between the set of 13 lines in $\tilde{Z}$ thus defined and that of the hypersurfaces in $C_{E_{7}}$ intersecting both $Y\left(M_{4, A}\right)$ and $Y\left(M_{4, B}\right)$. The result is given in Table 5. All the hypersurfaces of the $1 \mathrm{st}-4$ th kinds intersecting $Y\left(M_{4, B}\right)$ (respectively $Y\left(M_{4, A}\right)$ ) belong to the column whose heading is $Y\left(M_{4, B}\right)$ (respectively $Y\left(M_{4, A}\right)$ ).

In the sequel, $\rho$ denotes the map in (15). It is easy to show that $\rho\left(Y\left(M_{4, B}\right)\right) \simeq$ $Y\left(M_{4, B}\right)$ and $\rho\left(Y\left(M_{4, A}\right)\right) \simeq \mathbf{T}_{2}$. In the fourth column of Table 5 , we give a natural 
correspondence between the set of hypersurfaces in $C_{E_{7}}$ intersecting $M_{4, A}$ and that of hypersurfaces $\mathbf{T}_{2}(i j)$ in $\mathbf{T}_{2}$. We denote by $Y_{56}^{*}$ the image of $Y_{56} \cap Y\left(M_{4, A}\right)$ by $\rho$. It is easy to see that $Y_{56}^{*}$ is a curve in $\mathbf{T}_{2}$ passing through two points $\mathbf{T}_{2}(25) \cap \mathbf{T}_{2}(34)$, $\mathbf{T}_{2}(23) \cap \mathbf{T}_{2}(45)$ and intersecting two lines $\rho\left(Y_{12} \cap Y\left(M_{4, A}\right)\right), \rho\left(Y_{347} \cap Y\left(M_{4, A}\right)\right)$.

(Y.5) The case $Y\left(M_{5}\right)$. By virtue of $Y\left(M_{5}\right)=Y\left(M_{4, A}\right) \cap Y\left(M_{4, B}\right)$ and Table 5, we conclude that hypersurfaces of $C_{E_{7}}$ intersecting $Y\left(M_{5}\right)$ are

$$
Y_{12}, \quad Y_{347}, \quad W_{56,134}, W_{56,234} \text {. }
$$

(Y.6) The cases $Y\left(M_{6}\right)$ and $Y\left(M_{7}\right)$. From Table 5, we easily see that the intersection $Y\left(M_{6}\right)=\cap_{\beta \in M_{6}} Y_{\beta}$ consists of a point and the intersection $Y\left(M_{7}\right)=\cap_{\beta \in M_{7}} Y_{\beta}$ is empty.

\section{Hypersurfaces corresponding to subroot systems of type $E_{6}$}

The purpose of this section is to study hypersurfaces of $C_{E_{7}}$ invariant under $W\left(E_{6}\right)$ action (cf. [14, $\S 4,(4.15 .10)]$ ). If $\Delta_{1}$ is a subroot system of type $E_{6}$ in $\Delta$, it is easy to show that $Y_{\triangle, D_{4}}\left(\Delta_{1}\right)$ is a hypersurface of $C_{E_{7}}$. Such a hypersurface is said to be of the 5th kind. As was pointed out in [14, (4.15.10)], on the contrary to those of the 1st4th kinds, each hypersurface of the 5th kind has a non-empty intersection with $C_{E_{7}}^{\prime}$. In the sequel, we identify the root system $\Delta\left(E_{6}\right)$ of type $E_{6}$ with the subroot system of $\Delta$ whose simple roots are $\gamma_{12}, \gamma_{123}, \gamma_{23}, \gamma_{34}, \gamma_{45}, \gamma_{56}$ unless otherwise stated. We also identify the Weyl group $W\left(E_{6}\right)$ with the subgroup of $W\left(E_{7}\right)$ generated by the reflections with respect to the roots of $\Delta\left(E_{6}\right)$. As a basic property of hypersurfaces of the 5th kind, we show an identification of such hypersurfaces with the cross ratio variety $C\left(\Delta\left(E_{6}\right),\left\{A_{3}, D_{4}\right\}\right)$.

LEMMA 6. $Y_{\Delta, D_{4}}\left(\Delta\left(E_{6}\right)\right) \simeq C\left(\Delta\left(E_{6}\right),\left\{A_{3}, D_{4}\right\}\right)$.

Proof. There are 45 subroot systems of type $D_{4}$ and 270 subroot systems of type $A_{3}$ in $\Delta\left(E_{6}\right)$. If $\Delta_{1}$ is a subroot system of type $A_{3}$ in $\Delta\left(E_{6}\right)$, there is a unique subroot system of type $D_{4}$ in $\Delta$ containing $\Delta_{1}$. These combined with the definitions of $Y_{\Delta, D_{4}}\left(\Delta\left(E_{6}\right)\right)$ and $C\left(\Delta\left(E_{6}\right),\left\{A_{3}, D_{4}\right\}\right)$ imply the lemma.

We are going to characterize $Y_{\Delta, D_{4}}\left(\Delta\left(E_{6}\right)\right)$ in terms of configurations of seven points in $\mathbf{P}^{2}$. To accomplish this, we need some preparations. We take seven points $P_{1}, \ldots, P_{7}$ in $\mathbf{P}^{2}$ as in Section 3, (14). It is easy to see that for each $i(=1,2, \ldots, 7)$, there is a unique cubic curve $C\left(\left\{P_{1}, \ldots, P_{7}\right\} ; P_{i}\right)$ in $\mathbf{P}^{2}$ passing through $P_{1}, \ldots, P_{7}$ such that $P_{i}$ is a double point (cf. [7,8]). Let $Y\left(\left\{P_{1}, \ldots, P_{7}\right\} ; P_{i}\right)$ 
be a subset of $\mathbf{P}_{0}(2,7)$ consisting of $P G L(3)$-orbits of $X=\left(P_{1}, P_{2}, \ldots, P_{7}\right)$ such that $C\left(\left\{P_{1}, \ldots, P_{7}\right\} ; P_{i}\right)$ has a cusp at $P_{7}$ (cf. [7]).

Then the main purpose of this section is to prove the following.

Theorem 2. $Y\left(\left\{P_{1}, \ldots, P_{7}\right\} ; P_{7}\right)=Y_{\Delta, D_{4}}\left(\Delta\left(E_{6}\right)\right) \cap \mathbf{P}_{0}(2,7)$.

The basic idea of the proof employed here is to compare the defining equation of $Y_{\Delta, D_{4}}\left(\Delta_{1}\right)$ with that of $Y\left(\left\{P_{1}, \ldots, P_{7}\right\} ; P_{7}\right)$. Noting the action of $W\left(E_{7}\right)$ on both $\Delta$ and $\mathbf{P}_{0}(2,7)$, we find that the claim of the theorem is equivalent to the following:

$$
Y\left(\left\{P_{1}, \ldots, P_{7}\right\} ; P_{1}\right)=Y_{\Delta, D_{4}}\left(\Delta\left(E_{6}\right)^{\prime}\right) \cap \mathbf{P}_{0}(2,7),
$$

where $\Delta\left(E_{6}\right)^{\prime}=\left\langle\gamma_{23}, \gamma_{234}, \gamma_{34}, \gamma_{45}, \gamma_{56}, \gamma_{16}\right\rangle \cap \Delta\left(E_{7}\right)$ which is isomorphic to $\Delta\left(E_{6}\right)$.

We first find when the cubic curve $C\left(\left\{P_{1}, \ldots, P_{7}\right\} ; P_{1}\right)$ has a cusp at $P_{1}$. Take $\xi=\left(\xi_{1}: \xi_{2}: \xi_{3}\right)$ as a homogeneous coordinate of $\mathbf{P}^{2}$ and let

$$
F\left(\xi_{1}, \xi_{2}, \xi_{3}\right)=0
$$

be a defining equation of $C\left(\left\{P_{1}, \ldots, P_{7}\right\} ; P_{1}\right)$. Since our curve passes through $P_{1}, \ldots, P_{7}$, we have

$$
F\left(P_{j}\right)=0, \quad j=1, \ldots, 7 .
$$

Since the curve also has a cusp at $P_{1}$,

$$
\begin{gathered}
\frac{\partial F}{\partial \xi_{i}}\left(P_{1}\right)=0, \quad i=1,2,3, \\
\frac{\partial^{2} F}{\partial \xi_{2}^{2}}\left(P_{1}\right) \frac{\partial^{2} F}{\partial \xi_{3}^{2}}\left(P_{1}\right)-\left(\frac{\partial^{2} F}{\partial \xi_{2} \partial \xi_{3}}\left(P_{1}\right)\right)^{2}=0 .
\end{gathered}
$$

Put

$$
\begin{aligned}
F= & c_{1} \xi_{1}^{3}+c_{2} \xi_{2}^{3}+c_{3} \xi_{3}^{3}+c_{4} \xi_{1}^{2} \xi_{2}+c_{5} \xi_{1}^{2} \xi_{3}+c_{6} \xi_{1} \xi_{2}^{2}+c_{7} \xi_{2}^{2} \xi_{3}+c_{8} \xi_{1} \xi_{3}^{2} \\
& +c_{9} \xi_{2} \xi_{3}^{2}+c_{10} \xi_{1} \xi_{2} \xi_{3} .
\end{aligned}
$$

Then equations (17)-(19) are equivalent to

$$
\left\{\begin{array}{l}
c_{1}=c_{2}=c_{3}=c_{4}=c_{5}=0 \\
c_{6}+c_{7}+c_{8}+c_{9}+c_{10}=0 \\
c_{6} x_{1}^{2}+c_{7} x_{1}^{2} y_{1}+c_{8} y_{1}^{2}+c_{9} x_{1} y_{1}^{2}+c_{10} x_{1} y_{1}=0 \\
c_{6} x_{2}^{2}+c_{7} x_{2}^{2} y_{2}+c_{8} y_{2}^{2}+c_{9} x_{2} y_{2}^{2}+c_{10} x_{2} y_{2}=0 \\
c_{6} x_{3}^{2}+c_{7} x_{3}^{2} y_{3}+c_{8} y_{3}^{2}+c_{9} x_{3} y_{3}^{2}+c_{10} x_{3} y_{3}=0
\end{array}\right.
$$


and

$$
4 c_{6} c_{8}-c_{10}^{2}=0
$$

Using vectors

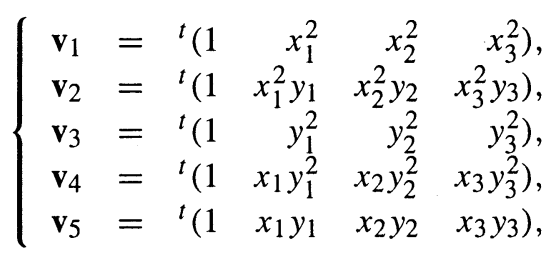

we define polynomials $\delta_{6}, \ldots, \delta_{10}$ of $x, y$ by

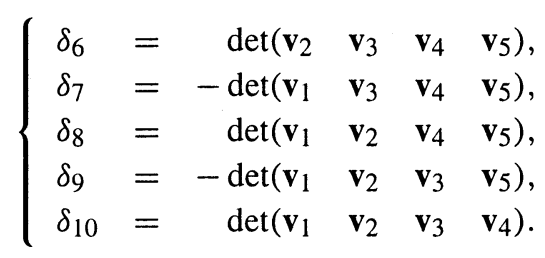

It is clear from the definition that if $\delta_{j} \neq 0$ for some $j=6, \ldots, 10$, the ratio of $c_{6}, \ldots, c_{10}$ is uniqely determined. This actually holds by the lemma below which is shown by direct computation.

LEMma 7. If $(x, y) \in \mathbf{C}^{6}-S\left(A_{6}\right)$, then $\delta_{j}(x, y) \neq 0$ for some $j \in\{6,7,8,9,10\}$.

Moreover, applying Cramer's formula to the system of equations (20) for $c_{6}, \ldots, c_{10}$, we find that

$$
\frac{c_{6}}{\delta_{6}}=\frac{c_{7}}{\delta_{7}}=\frac{c_{8}}{\delta_{8}}=\frac{c_{9}}{\delta_{9}}=\frac{c_{10}}{\delta_{10}} .
$$

By virtue of equations (21) and (24), we obtain the following lemma.

Lemma 8. The subvariety $Y\left(\left\{P_{1}, \ldots, P_{7}\right\} ; P_{1}\right)$ is the hypersurface of $\mathbf{P}_{0}(2,7)$ defined by the equation

$$
4 \delta_{6} \delta_{8}-\delta_{10}^{2}=0
$$

We now consider the hypersurface $Z=\sigma_{123} \sigma_{145}\left(Y\left(\left\{P_{1}, \ldots, P_{7}\right\} ; P_{1}\right)\right)$, where $\sigma_{123}$ and $\sigma_{145}$ denote the images of the reflections $s_{123}$ and $s_{145}$ by the surjective homomorphism $p_{W\left(E_{7}\right)}: W\left(E_{7}\right) \rightarrow W_{7}$ introduced in Section 2, respectively. We determine the defining equation of $Z$. 
LEMMA 9. The hypersurface $Z$ is defined by the equation

$$
\left(q_{1}+q_{2}+q_{3}\right)^{2}-4 q_{1} q_{2}=0
$$

where

$$
\left\{\begin{array}{l}
q_{1}=x_{1}\left(1-y_{1}\right)\left(y_{2}-y_{3}\right), \\
q_{2}=y_{1}\left(1-x_{1}\right)\left(x_{2}-x_{3}\right), \\
q_{3}=\left(x_{1}-y_{1}\right)\left(x_{2} y_{3}-x_{3} y_{2}\right) .
\end{array}\right.
$$

Proof. The birational transformation $\sigma_{123} \sigma_{145}$ is given by

$$
\begin{aligned}
\sigma_{123} \sigma_{145} & :\left(x_{1}, x_{2}, x_{3}, y_{1}, y_{2}, y_{3}\right) \\
& \longrightarrow\left(\frac{1}{y_{1}}, \frac{\psi_{2}}{x_{2} y_{1} \varphi_{2}}, \frac{\psi_{3}}{x_{3} y_{1} \varphi_{3}}, \frac{1}{x_{1}}, \frac{\psi_{2}}{x_{1} y_{2} \varphi_{2}}, \frac{\psi_{3}}{x_{1} y_{3} \varphi_{3}}\right),
\end{aligned}
$$

where

$$
\begin{aligned}
& \varphi_{2}=x_{1}-x_{2}-y_{1}+y_{2}+x_{2} y_{1}-x_{1} y_{2}, \\
& \varphi_{3}=x_{1}-x_{3}-y_{1}+y_{3}+x_{3} y_{1}-x_{1} y_{3}, \\
& \psi_{2}=x_{1} y_{2}-x_{2} y_{1}+x_{1} x_{2}\left(y_{1}-y_{2}\right)-y_{1} y_{2}\left(x_{1}-x_{2}\right), \\
& \psi_{3}=x_{1} y_{3}-x_{3} y_{1}+x_{1} x_{3}\left(y_{1}-y_{3}\right)-y_{1} y_{3}\left(x_{1}-x_{3}\right) .
\end{aligned}
$$

Moreover,

where

$$
\left\{\begin{array}{l}
\delta_{6} \circ \sigma_{123} \sigma_{145}=\frac{\psi_{2}^{2} \psi_{3}^{2} \delta_{0}}{x_{1}^{5} x_{2}^{2} x_{3}^{2} y_{1}^{5} y_{2}^{2} y_{3}^{2} \varphi_{2}^{3} \varphi_{3}^{3}} \cdot \delta_{6}^{\prime}, \\
\delta_{8} \circ \sigma_{123} \sigma_{145}=\frac{\psi_{2}^{2} \psi_{3}^{2} \delta_{0}}{x_{1}^{5} x_{2}^{2} x_{3}^{2} y_{1}^{5} y_{2}^{2} y_{3}^{2} \varphi_{2}^{3} \varphi_{3}^{3}} \cdot \delta_{8}^{\prime}, \\
\delta_{10 \circ \sigma_{123} \sigma_{145}}=\frac{\psi_{2}^{2} \psi_{3}^{2} \delta_{0}}{x_{1}^{5} x_{2}^{2} x_{3}^{2} y_{1}^{5} y_{2}^{2} y_{3}^{2} \varphi_{2}^{3} \varphi_{3}^{3}} \cdot \delta_{10}^{\prime}
\end{array}\right.
$$

$$
\begin{aligned}
\delta_{0} & =\left(1-x_{1}\right)\left(1-y_{1}\right)\left(x_{2}-y_{2}\right)\left(x_{3}-y_{3}\right)\left(x_{1} y_{2}-x_{2} y_{1}\right)\left(x_{1} y_{3}-x_{3} y_{1}\right), \\
\delta_{6}^{\prime} & =-y_{1}\left(x_{2}-x_{3}\right)\left(1-y_{1}\right), \\
\delta_{8}^{\prime} & =-x_{1}\left(1-x_{1}\right)\left(y_{2}-y_{3}\right), \\
\delta_{10}^{\prime} & =x_{1}\left(1-y_{1}\right)\left(y_{2}-y_{3}\right)+y_{1}\left(1-x_{1}\right)\left(x_{2}-x_{3}\right)+\left(x_{1}-y_{1}\right)\left(x_{2} y_{3}-x_{3} y_{2}\right) .
\end{aligned}
$$

Then we find from Lemma 8 that $Z$ is defined by

$$
4 \delta_{6}^{\prime} \delta_{8}^{\prime}-\delta_{10}^{\prime 2}=0 \text {. }
$$

This equation is equivalent to (26) and the lemma follows. 
We next find a defining equation of $Y_{\Delta, D_{4}}\left(s_{123} s_{145} \Delta\left(E_{6}\right)^{\prime}\right)$. A straightforward computation leads to the following.

LEMMA 10.

$$
\begin{aligned}
1-x_{1} & =\frac{h_{12} h_{123} h_{345} h_{45}}{h_{134} h_{14} h_{235} h_{25}}, \\
x_{2}-x_{3} & =\frac{h_{12} h_{123} h_{234} h_{24} h_{367} h_{67}}{h_{134} h_{14} h_{236} h_{237} h_{26} h_{27}}, \\
1-y_{1} & =\frac{h_{123} h_{13} h_{245} h_{45}}{h_{124} h_{14} h_{235} h_{35}}, \\
y_{2}-y_{3} & =\frac{h_{123} h_{13} h_{234} h_{267} h_{34} h_{67}}{h_{124} h_{14} h_{236} h_{237} h_{36} h_{37}}, \\
x_{1}-y_{1} & =\frac{h_{123} h_{145} h_{15} h_{23} h_{234} h_{45}}{h_{124} h_{134} h_{14} h_{235} h_{25} h_{35}}, \\
x_{2} y_{3}-x_{3} y_{2} & =-\frac{h_{123} h_{16} h_{167} h_{17} h_{23} h_{234}^{2} h_{24} h_{34} h_{67}}{h_{124} h_{134} h_{14}^{2} h_{236} h_{237} h_{26} h_{27} h_{36} h_{37}} .
\end{aligned}
$$

Putting $\alpha_{1}^{\prime}=\gamma_{23}, \alpha_{2}^{\prime}=\gamma_{14}, \alpha_{3}^{\prime}=\gamma_{25}, \alpha_{4}^{\prime}=\gamma_{45}, \alpha_{5}^{\prime}=\gamma_{146}, \alpha_{6}^{\prime}=\gamma_{7}, \alpha_{7}^{\prime}=\gamma_{6}$, we find that $\alpha_{1}^{\prime}, \alpha_{2}^{\prime}, \ldots, \alpha_{6}^{\prime}$ (respectively $\alpha_{1}^{\prime}, \alpha_{2}^{\prime}, \ldots, \alpha_{7}^{\prime}$ ) form a system of simple roots in $s_{123} s_{145} \Delta\left(E_{6}\right)^{\prime}$ (respectively $\Delta$ ). Moreover, we put

$$
\zeta_{i}=\left\langle\alpha_{j}^{\prime}, t\right\rangle, \quad j=1,2, \ldots, 7
$$

for $t \in E_{\mathbf{C}}$. Since each of $h_{i}, h_{i j}, h_{i j k}$ is expressed as a linear combination of $\zeta_{1}, \ldots, \zeta_{7}$, we regard each of them as a linear function of $\zeta_{1}, \ldots, \zeta_{7}$ in the subsequent argument. If $\gamma$ is a root of $s_{123} s_{145} \Delta\left(E_{6}\right)^{\prime}, \gamma$ is a linear combination of $\alpha_{1}^{\prime}, \ldots, \alpha_{6}^{\prime}$ and therefore $\langle\gamma, t\rangle\left(t \in E_{\mathbf{C}}\right)$ is a linear function of $\zeta_{1}, \ldots, \zeta_{6}$ and is independent of $\zeta_{7}$. For $\gamma \in s_{123} s_{145} \Delta\left(E_{6}\right)^{\prime}$, we write

$$
\gamma=\sum_{j=1}^{6} n_{\gamma, j} \alpha_{j}^{\prime} \quad\left(n_{\gamma, j} \in \mathbf{Z}\right) .
$$

Using this notation, we define

$$
F_{\mathbf{C}}^{\prime}=\left\{\left(\zeta_{1}, \ldots, \zeta_{6}\right) \in \mathbf{C}^{6} ; \sum_{j=1}^{6} n_{\gamma, j} \zeta_{j} \neq 0\left(\forall \gamma \in s_{123} s_{145} \Delta\left(E_{6}\right)^{\prime}\right)\right\} .
$$

Then $\left(\zeta_{1}, \ldots, \zeta_{7}\right)$ is regarded as a coordinate of $E_{\mathbf{C}}$ and we may take

$$
\zeta=\left(\zeta_{1}: \cdots: \zeta_{6}: \zeta_{7}\right)
$$


as a homogeneous coordinate of $\mathbf{P}^{6}$. Since, from the definition, $Z \cap\left\{\zeta_{7} \neq 0\right\}$ is Zariski open in $Z$, we may restrict our attention to an open subset of $\mathbf{P}^{6}$ defined by $\zeta_{7} \neq 0$. Now we write

$$
\zeta_{j}=\zeta_{j}^{\prime} \tau \quad(j=1, \ldots, 6)
$$

Then

$$
\tilde{\zeta}=\left(\left(\zeta_{1}^{\prime}: \cdots: \zeta_{6}^{\prime}\right), \tau\right)
$$

is regarded as a local coordinate of the open subset defined by $\zeta_{7} \neq 0$ in $\mathbf{P}^{6}$. Noting this, we define rational functions $\tilde{x}_{j}, \tilde{y}_{j}$ of $\tilde{\zeta}$ by

$$
\tilde{x}_{j}(\tilde{\zeta})=x_{j}(t), \quad \tilde{y}_{j}(\tilde{\zeta})=y_{j}(t) \quad(j=1,2,3)
$$

and put

$$
u_{j}\left(\zeta_{1}^{\prime}: \cdots: \zeta_{6}^{\prime}\right)=\lim _{\tau \rightarrow 0} \tilde{x}_{j}(\tilde{\zeta}), \quad v_{j}\left(\zeta_{1}^{\prime}: \cdots: \zeta_{6}^{\prime}\right)=\lim _{\tau \rightarrow 0} \tilde{y}_{j}(\tilde{\zeta}) \quad(j=1,2,3) .
$$

LEMMA 11.

$$
\begin{aligned}
\frac{u_{1}\left(1-v_{1}\right)\left(v_{2}-v_{3}\right)}{\left(u_{1}-v_{1}\right)\left(u_{2} v_{3}-u_{3} v_{2}\right)} & =-\frac{\left(\zeta_{1}^{\prime}+\zeta_{2}^{\prime}-\zeta_{3}^{\prime}+\zeta_{4}^{\prime}\right)^{2}}{\left(\zeta_{2}^{\prime}-\zeta_{3}^{\prime}+\zeta_{4}^{\prime}\right)^{2}} \\
\frac{v_{1}\left(1-u_{1}\right)\left(u_{2}-u_{3}\right)}{\left(u_{1}-v_{1}\right)\left(u_{2} v_{3}-u_{3} v_{2}\right)} & =-\frac{\zeta_{1}^{\prime 2}}{\left(\zeta_{2}^{\prime}-\zeta_{3}^{\prime}+\zeta_{4}^{\prime}\right)^{2}} .
\end{aligned}
$$

Proof. By virtue of (27) and Lemma 10, we find that

$$
\begin{aligned}
& q_{1}=\frac{h_{13}^{2} h_{135} h_{245} h_{267}}{h_{124} h_{235} h_{36} h_{37}} \cdot q_{0}, \\
& q_{2}=\frac{h_{12}^{2} h_{125} h_{345} h_{367}}{h_{134} h_{235} h_{26} h_{27}} \cdot q_{0}, \\
& q_{3}=-\frac{h_{145} h_{23}^{2} h_{234} h_{16} h_{17} h_{167}}{h_{124} h_{134} h_{26} h_{27} h_{36} h_{37}} \cdot q_{0},
\end{aligned}
$$

where

$$
q_{0}=\frac{h_{123}^{2} h_{15} h_{24} h_{34} h_{234}^{2} h_{45} h_{67}}{h_{14}^{3} h_{124} h_{134} h_{25} h_{35} h_{235} h_{236} h_{237}} .
$$

Using these relations and the definitions of $h_{i j}, h_{i j k}$, we obtain the lemma.

LEMMA 12. If $(x, y) \in \mathbf{P}_{0}(2,7)$ is contained in $W=Y_{\Delta, D_{4}}\left(s_{123} s_{145} \Delta\left(E_{6}\right)^{\prime}\right)$, then

$$
\left(q_{1}+q_{2}+q_{3}\right)^{2}-4 q_{1} q_{2}=0 .
$$


Proof. We first define $W^{\prime}$ by

$$
\begin{array}{r}
W^{\prime}=\left\{\left(u_{1}, u_{2}, u_{3}, v_{1}, v_{2}, v_{3}\right) ; u_{j}\left(\zeta_{1}^{\prime}: \cdots: \zeta_{6}^{\prime}\right)=\lim _{\tau \rightarrow 0} \tilde{x}_{j}(\tilde{\zeta}),\right. \\
\left.v_{j}\left(\zeta_{1}^{\prime}: \cdots: \zeta_{6}^{\prime}\right)=\lim _{\tau \rightarrow 0} \tilde{y}_{j}(\tilde{\zeta})\left(\exists\left(\zeta_{1}^{\prime}, \ldots, \zeta_{6}^{\prime}\right) \in F_{\mathbf{C}}^{\prime}\right)\right\} .
\end{array}
$$

From the definition, we find that $W$ is the Zariski closure of $W^{\prime} \cap \mathbf{P}_{0}(2,7)$. Then, if $(x, y) \in W^{\prime}$, we find that

$$
x_{j}=u_{j}\left(\zeta_{1}^{\prime}: \cdots: \zeta_{6}^{\prime}\right), \quad y_{j}=v_{j}\left(\zeta_{1}^{\prime}: \cdots: \zeta_{6}^{\prime}\right) \quad(j=1,2,3)
$$

for some $\left(\zeta_{1}^{\prime}, \ldots, \zeta_{6}^{\prime}\right) \in F_{\mathrm{C}}^{\prime}$. This combined with Lemma 11 implies that

$$
\begin{aligned}
\frac{x_{1}\left(1-y_{1}\right)\left(y_{2}-y_{3}\right)}{\left(x_{1}-y_{1}\right)\left(x_{2} y_{3}-x_{3} y_{2}\right)} & =-\frac{\left(\zeta_{1}^{\prime}+\zeta_{2}^{\prime}-\zeta_{3}^{\prime}+\zeta_{4}^{\prime}\right)^{2}}{\left(\zeta_{2}^{\prime}-\zeta_{3}^{\prime}+\zeta_{4}^{\prime}\right)^{2}}, \\
\frac{y_{1}\left(1-x_{1}\right)\left(x_{2}-x_{3}\right)}{\left(x_{1}-y_{1}\right)\left(x_{2} y_{3}-x_{3} y_{2}\right)} & =-\frac{\zeta_{1}^{\prime 2}}{\left(\zeta_{2}^{\prime}-\zeta_{3}^{\prime}+\zeta_{4}^{\prime}\right)^{2}} .
\end{aligned}
$$

These identities imply that

$$
\begin{aligned}
\left\{x_{1}(1\right. & \left.\left.-y_{1}\right)\left(y_{2}-y_{3}\right)+y_{1}\left(1-x_{1}\right)\left(x_{2}-x_{3}\right)+\left(x_{1}-y_{1}\right)\left(x_{2} y_{3}-x_{3} y_{2}\right)\right\}^{2} \\
& -4 x_{1} y_{1}\left(1-x_{1}\right)\left(x_{2}-x_{3}\right)\left(1-y_{1}\right)\left(y_{2}-y_{3}\right) \\
= & \left(x_{1}-y_{1}\right)^{2}\left(x_{2} y_{3}-x_{3} y_{2}\right)^{2} \\
& \quad \times \frac{\left\{\left(\zeta_{1}^{\prime}+\zeta_{2}^{\prime}-\zeta_{3}^{\prime}+\zeta_{4}^{\prime}\right)^{2}+\zeta_{1}^{\prime 2}-\left(\zeta_{2}^{\prime}-\zeta_{3}^{\prime}+\zeta_{4}^{\prime}\right)^{2}\right\}^{2}-4 \zeta_{1}^{\prime 2}\left(\zeta_{1}^{\prime}+\zeta_{2}^{\prime}-\zeta_{3}^{\prime}+\zeta_{4}^{\prime}\right)^{2}}{\left(\zeta_{2}^{\prime}-\zeta_{3}^{\prime}+\zeta_{4}^{\prime}\right)^{2}} \\
= & 0 .
\end{aligned}
$$

Therefore

$$
\begin{gathered}
\left\{x_{1}\left(1-y_{1}\right)\left(y_{2}-y_{3}\right)+y_{1}\left(1-x_{1}\right)\left(x_{2}-x_{3}\right)+\left(x_{1}-y_{1}\right)\left(x_{2} y_{3}-x_{3} y_{2}\right)\right\}^{2} \\
-4 x_{1} y_{1}\left(1-x_{1}\right)\left(x_{2}-x_{3}\right)\left(1-y_{1}\right)\left(y_{2}-y_{3}\right)=0
\end{gathered}
$$

which is equivalent to

$$
\left(q_{1}+q_{2}+q_{3}\right)^{2}-4 q_{1} q_{2}=0 .
$$

We have thus proved the lemma.

Proof of Theorem 2. We retain the notation above without any comment. By virtue of Lemmas 9 and 12, we find that

$$
Y_{\Delta, D_{4}}\left(s_{123} s_{145} \Delta\left(E_{6}\right)\right) \cap C^{\prime}\left(\Delta, E_{7}\right)=Y_{\Delta, D_{4}}\left(s_{123} s_{145} \Delta\left(E_{6}\right)\right) \cap \mathbf{P}_{0}(2,7) \subset Z .
$$


Since it is clear from the definition that both $Y_{\Delta, D_{4}}\left(s_{123} s_{145} \Delta\left(E_{6}\right)\right) \cap C^{\prime}\left(\Delta, E_{7}\right)$ and $Z$ are five-dimensional irreducible varieties, we conclude that

$$
Y_{\Delta, D_{4}}\left(s_{123} s_{145} \Delta\left(E_{6}\right)\right) \cap C^{\prime}\left(\Delta, E_{7}\right)=Z .
$$

This implies the theorem.

Remark 6. (cf. [15]) It is an easy consequence of Theorem 2 that the isotropy group of the hypersurface $Y\left(\left\{P_{1}, \ldots, P_{7}\right\} ; P_{7}\right)$ in $W\left(E_{7}\right)$ coincides with $\operatorname{Aut}_{W\left(E_{7}\right)}\left(W\left(E_{6}\right)\right) \simeq$ $W\left(E_{6}\right) \times \mathbf{Z}_{2}$.

Let $\omega_{j}$ be the fundamental weight of $\Delta\left(E_{7}\right)$ such that $\left\langle\omega_{j}, \alpha_{k}\right\rangle=\delta_{j k}$. Then $\omega_{7}$ belongs to the set $\Omega_{56}$ of weights of the 56-dimensional irreducible representation of the simple Lie algebra of type $E_{7}$. There is a two-to-one correspondence of $\Omega_{56}$ to the $W\left(E_{7}\right)$-orbit of $Y\left(\left\{P_{1}, \ldots, P_{7}\right\} ; P_{7}\right)$. The concrete correspondence is given as follows. First $\pm \omega_{7}$ correspond to $Y\left(\left\{P_{1}, \ldots, P_{7}\right\} ; P_{7}\right)$. The weights $\pm s \cdot \omega_{7}$ for $s \in W\left(E_{7}\right)$ correspond to $s \cdot Y\left(\left\{P_{1}, \ldots, P_{7}\right\} ; P_{7}\right)$. In particular, if $s \cdot \omega_{7}=$ $\varepsilon_{1}+\varepsilon_{2}+\varepsilon_{3}+\varepsilon_{4}-\varepsilon_{5}-\varepsilon_{6}$, then $s \cdot Y\left(\left\{P_{1}, \ldots, P_{7}\right\} ; P_{7}\right)$ coincides with the hypersurface defined by (26).

Acknowledgement. The author is indebted to the referee for a careful reading of the paper and for kind advice.

\section{REFERENCES}

[1] N. Bourbaki. Groupes et Algèbres de Lie, Chaps. 4, 5, 6. Herman, Paris 1968.

[2] I. Dolgachev and D. Ortland. Point sets in projective spaces and theta functions. Astérisque 165 (1988).

[3] T. Fukui and J. Sekiguchi. A remark on labelled 8 lines on the real projective plane. Reports of Fac. Sci., Himeji Inst. Tech. 8 (1997), 1-11.

[4] T. Fukui and J. Sekiguchi. Eight lines arrangements on the real projective plane and the root system of type $E_{8}$. Proc. Third Asian Tech. Conference in Math., August 24-28, 1998, Tsukuba, 377-388.

[5] B. Grünbaum. Convex Polytopes. Interscience, 1967.

[6] B. Hunt. A remarkable quintic fourfold in $\mathbf{P}^{5}$ and its dual variety (Update: 7.1.1992). Preprint.

[7] E. Looijenga. Moduli spaces of marked del Pezzo surfaces. Appendix in [9].

[8] Yu. I. Manin. Cubic Forms, Algebra, Geometry, Arithmetic. North-Holland, Amsterdam, 1974.

[9] I. Naruki. Cross ratio variety as a moduli space of cubic surfaces. Proc. London Math. Soc. 45 (1982), 1-30.

[10] I. Naruki and J. Sekiguchi. A modification of Cayley's family of cubic surfaces and birational action of $W\left(E_{6}\right)$ over it. Proc. Japan Acad. 56 Ser. A (1980), 122-125.

[11] T. Oda. The canonical compactification of the configuration space of the pure braid group of $\mathbf{P}^{1}$. Preprint. 
[12] J. Sekiguchi. The configuration space of 6 points in $\mathbf{P}^{2}$, the moduli space of cubic surfaces and the Weyl group of type $E_{6}$. Sūrikaisekikenkyūsho Kōkyūroku 848 (1993), 74-85.

[13] J. Sekiguchi. The versal deformation of the $E_{6}$-singularity and a family of cubic surfaces. J. Math. Soc. Japan 46 (1994), 355-383.

[14] J. Sekiguchi. Cross ratio varieties for root systems. Kyushu J. Math. 48 (1994), 123-168.

[15] J. Sekiguchi. $W\left(E_{7}\right)$-invariant polynomial of degree 10 and 28 bitangents of plane quartic curves. Sūrikaisekikenkyūsho Kōkyūroku 920 (1995), 216-228.

[16] J. Sekiguchi. Cross ratio varieties for root systems of type $A$ and the Terada model. J. Math. Sci. Univ. Tokyo 3 (1996), 181-197.

[17] J. Sekiguchi. Geometry of 7 lines on the real projective plane and the root system of type $E_{7}$. Sūrikaisekikenkyūsho Kōkyūroku 986 (1997), 1-8.

[18] J. Sekiguchi. Configurations of seven lines on the real projective plane and the root system of type $E_{7}$. To appear in J. Math. Soc. Japan.

[19] J. Sekiguchi and T. Tanabata. Tetradiagrams for the root system of type $E_{7}$ and its application. Reports of Faculty of Science, Himeji Inst. Tech. 7 (1996), 1-10.

[20] J. Sekiguchi and M. Yoshida. $W\left(E_{6}\right)$-action on the configuration space of six points on the real projective plane. Kyushu J. Math. 51 (1997), 297-354.

[21] T. Shioda. Construction of elliptic curves with high rank via the invariants of the Weyl groups. J. Math. Soc. Japan 43 (1991), 673-719.

[22] T. Shioda. Plane quartics and Mordell-Weil lattices of type $E_{7}$. Comment. Math. Univ. St. Pauli 42 (1993), 61-79.

[23] T. Shioda. A uniform construction of the root lattices $E_{6}, E_{7}, E_{8}$ and their dual lattices. Proc. Japan Acad. 71 Ser. A (1995), 140-143.

[24] T. Terada. Fonction hypergéométriques $F_{1}$ et fonctions automorph I. J. Math. Soc. Japan 35 (1983), 451-475.

\author{
Jiro Sekiguchi \\ Department of Mathematics \\ Himeji Institute of Technology \\ Himeji 671-2201 \\ Japan \\ (E-mail:sekiguti@sci.himeji-tech.ac.jp)
}

